\title{
8 Ring-Resonator-Based Wavelength Filters
}

Douwe H. Geuzebroek and Alfred Driessen

\subsection{Introduction}

Microring resonators (MR) represent a class of filters with characteristics very similar to those of Fabry-Perot filters. However, they offer the advantage that the injected and reflected signals are separated in individual waveguides, and in addition, their design does not require any facets or gratings and is thus particularly simple. MRs evolved from the fields of fibre optic ring resonators and micron scale droplets [1-4]. Their inherently small size (with typical diameters in the range between several to tens of micrometres), their filter characteristics and their potential for being used in complex and flexible configurations make these devices particularly attractive for integrated optics or VLSI photonics applications [5-10].

MRs for filter applications, delay lines, as add/drop multiplexers, and modulators will be covered in detail in this chapter, while other applications such as in optical sensing, in spectroscopy or for coherent light generation (MR lasers) are outside the scope of this chapter.

This chapter focuses primarily on 4-port microrings, while 2-port devices will play a minor role here and are covered in more detail in Chap. 9. The present chapter starts with design considerations, the functional behaviour, and key characteristics of a single microring resonator and continues with the design of cascaded MRs allowing the implementation of higher order filters. Finally, complex devices like add-drop filters, tuneable dispersion compensators, all-optical wavelength converters, and tuneable cross-connects are treated. 


\subsection{Fundamentals of Microring Resonators}

\subsubsection{General Considerations}

The fundamental building blocks of microring-based devices are a microring plus one or two waveguides. In the former case this leads to two-port devices, which act as all-pass filters and introduce a wavelength-dependent phase shift only (lossless case). This property is exploited for the realization of dispersion compensators and will be illustrated in Sect. 8.6.5.

On the other hand, a microring resonator consisting of a ring plus two straight waveguides represents a 4-port structure, as illustrated in Fig. 8.1.

The ring (radius $R$ ) and the port waveguides are evanescently coupled and a fraction $\kappa_{1}$ of the incoming field is transferred to the ring. When the optical path-length of a roundtrip is a multiple of the effective wavelength, constructive interference occurs and light is 'built up' inside the ring: the MR is ON resonance. As a consequence, periodic fringes appear in the wavelength response at the output ports as shown in Fig. 8.2. At resonance the drop port shows maximum transmission since a fraction $\kappa_{2}$ of the builtup field inside the ring is coupled to this port. In the through port the ring exhibits a minimum at resonance. In the ideal case with equal coupling constants at resonance all the power is directed to the drop port. Light coupled back to the through port after a roundtrip experiences an additional $180^{\circ}$ phase shift with respect to the light coming directly from the in port, and as a consequence no light exits from the through port at resonance.

Before coming to a more detailed description of MRs it is worthwhile to introduce a number of key parameters of MRs.
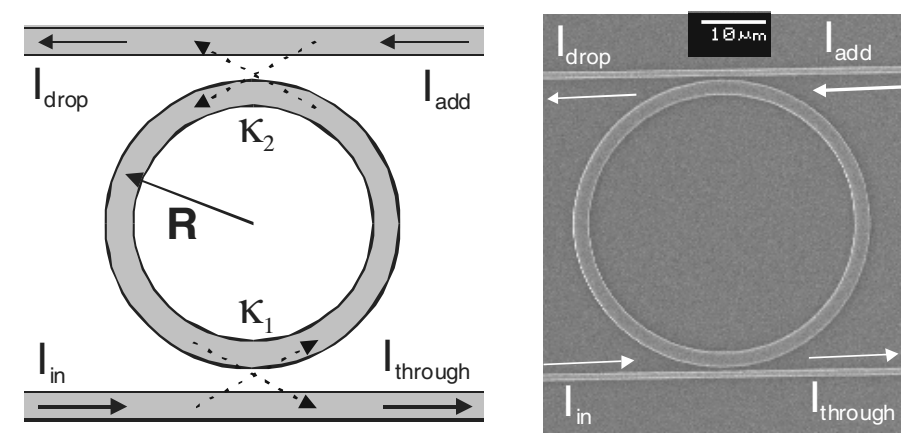

Fig. 8.1. Schematic drawing of a 4-port microring resonator (left) and SEM picture (right) with in- and output port waveguides 


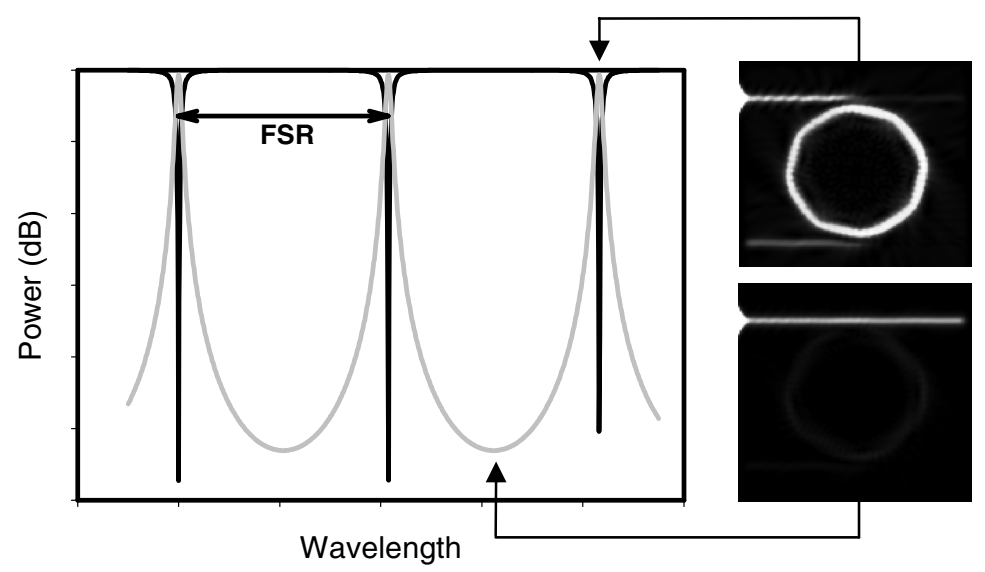

Fig. 8.2. Simulated response of a MR for the through- (black) and drop port (grey). Also shown is the Free Spectral Range, the distance between two consecutive fringes. At the right simulated fields of the MR are given; top: ON resonance, Bottom: OFF resonance

The difference in position between two consecutive resonant peaks, see Fig. 8.2, is called the Free Spectral Range and can be defined either in the frequency or wavelength domain $\left(F S R_{f}\right.$ or $F S R_{\lambda}$, respectively):

$$
F S R_{f}=\Delta f=\frac{c}{n_{g} 2 \pi R}, F S R_{\lambda}=\Delta \lambda \approx \frac{\lambda^{2}}{n_{g} 2 \pi R}
$$

The group index $n_{g}$ is defined as [9]

$$
n_{g}=n_{e f f}\left(f_{0}\right)+\left.f_{0} \frac{d n_{\text {eff }}}{d f}\right|_{f_{0}}=n_{e f f}\left(\lambda_{0}\right)+\left.\lambda_{0} \frac{d n_{e f f}}{d \lambda}\right|_{\lambda_{0}}
$$

Other parameters are: $n_{\text {eff }}$ is the effective refractive index, $R$ the radius of the ring, $\mathrm{c}$ the speed of light in vacuum and $f_{0}$ is defined by $f_{1,2}=f_{0} \pm \Delta f / 2$, with $f_{1}$ and $f_{2}$ two consecutive resonance frequencies.

Another important parameter of the MR is the Full Width at Half Maximum (FWHM) or 3-dB bandwidth, which is a measure for the bandwidth of the device. A quality measure of the microring is the Finesse $F$, which is the ratio between the FSR and the 3-dB bandwidth [11]

$$
\begin{aligned}
& F=\frac{F S R}{F W H M}=\frac{\pi\left(X_{1} X_{2}\right)^{1 / 4}}{1-\left(X_{1} X_{2}\right)^{1 / 2}} \\
& X_{i}=\sqrt{\left(1-\kappa_{i}^{2}\right)} e^{L_{r} \alpha}
\end{aligned}
$$


with $\alpha$ being the losses per length inside the ring and $L_{r}$ the length of the optical path in the ring. High finesse devices have a small FWHM and a strong intensity build-up in the ring when in resonance. Therefore high $F$ devices are suitable for applications were high intensities in the cavity are necessary like lasers or non-linear optical (NLO) devices. Instead of the finesse, the quality factor $Q$ can also be used as an absolute measure for the wavelength selectivity of the microring resonator according to:

$$
Q=\omega / \Delta \omega_{F W H M}=\lambda / \Delta \lambda_{F W H M}
$$

where $\Delta \omega_{F W H M}$ and $\Delta \lambda_{F W H M}$ are the 3-dB bandwidths in the frequency and wavelength domain, respectively.

The speed of high bit rate communication is limited by the bandwidth of the optical filters. As this bandwidth in high finesse MR devices is becoming increasingly small, only medium finesse devices $(F \sim 10-20)$ with relative large coupling constants $(\kappa \sim 0.4-0.6)$ are employed. The FWHM is determined by the coupling constants and the loss inside the ring according to $[12,13]$ :

$$
F W H M_{f}=\frac{c}{2 \pi R n_{g}}\left(\frac{\sqrt{X_{1} X_{2}}}{\pi \sqrt[4]{X_{1} X_{2}}}\right)
$$

\subsubsection{Bent Waveguides}

Bent waveguides exhibit radiation losses, which -for a given confinement of the guided wave- increase as the bend radius decreases. On the other hand, for a given bend radius, the bending losses decrease, as the confinement of the guided wave gets stronger, or alternatively spoken, the bending losses decrease, as the (effective) index contrast between the ring and its surrounding/substrate increases. In the following we will focus on high index contrast rings $(\Delta n>0.1)$, since this is a prerequisite for small footprint devices offering the required integration potential. $\Delta n$ is the difference of the effective indices of the ring waveguide and its surrounding, respectively.

In relation to the index contrast the term 'minimum bend radius' is used to define the lowest acceptable (technology-dependent!) radius keeping the bend losses sufficiently low. 'Sufficiently low' is dependent of the application and also related to the coupling fractions as this chapter will show. Typically one designs for a bending loss below $1 \mathrm{~dB}$ per $360^{\circ}$ roundtrip. The minimum bend radius as a function of effective index contrast is illustrated in Fig. 8.3. As can be seen, a high index contrast enables extremely 


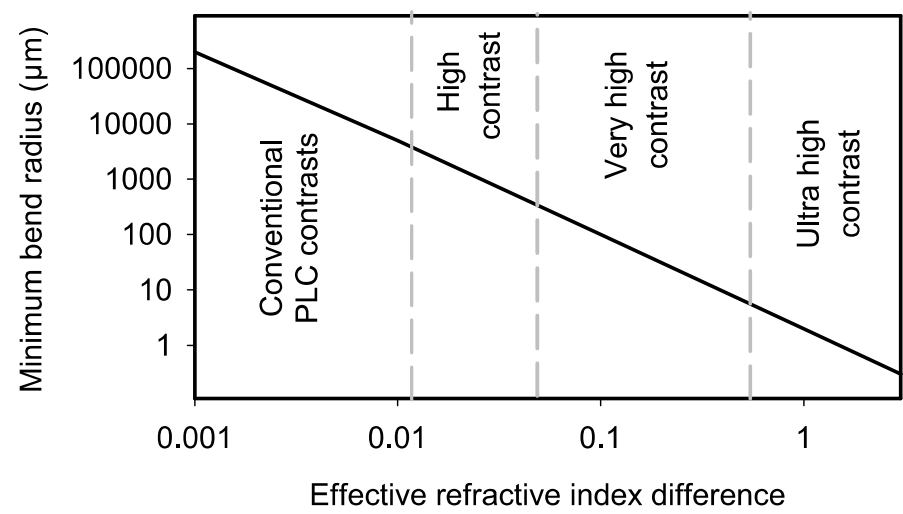

Fig. 8.3. Minimum bending radius $\left(<1 \mathrm{~dB} / 360^{\circ}\right)$ as a function of effective index difference

small dimensions and thus compact structures, but at the same time puts severe challenges to the fabrication technology. In addition, the stronger the confinement and the smaller the ring dimensions the more polarisationrelated issues become important as will be discussed in the next section.

\subsubsection{Polarisation Dependence}

Polarisation dependence is primarily determined by the waveguide characteristics (refractive index contrast, geometry [14]). However, even if a chosen geometry would exhibit low birefringence in a straight waveguide (i. e. the propagation constants for TE- and TM-polarized light are approximately the same) this is normally no longer true in bends or rings with small radii. In that case, the modes are no longer pure TE- or TM-like, the resonance wavelengths become polarisation dependent, and even polarisation conversion can occur [15-18].

If pure TE- and TM-like modes with equal coupling constants are assumed, the difference in resonance wavelength of the modes propagating inside the ring is

$$
\Delta \lambda_{c}=\frac{\lambda\left(n_{\text {eff }, T E}-n_{\text {eff }, T M}\right)}{n_{\text {eff }}}
$$

with $n_{\text {eff,TE }}$ and $n_{\text {eff,TM }}$ being the effective indices of the TE and TM mode inside the ring.

In general, in addition to the propagation constants the coupling constant between the ring and waveguides are also different for TE- and TM-polarisation. Nevertheless, polarisation independence can in principle be 
achieved by carefully balancing material- and geometry dependent effects [19]. In reality this is restricted to material with low index contrast [20,21] and it requires the potential to keep technological tolerances extremely tight. An alternative, although more complex, solution is polarisation diversity [22].

\subsection{Design of Single Microrings}

\subsubsection{Evaluation of Parameters}

One frequently chosen way of modelling the response of a single MR is the use of a scattering matrix $[23,24]$ as illustrated in Fig. 8.4.
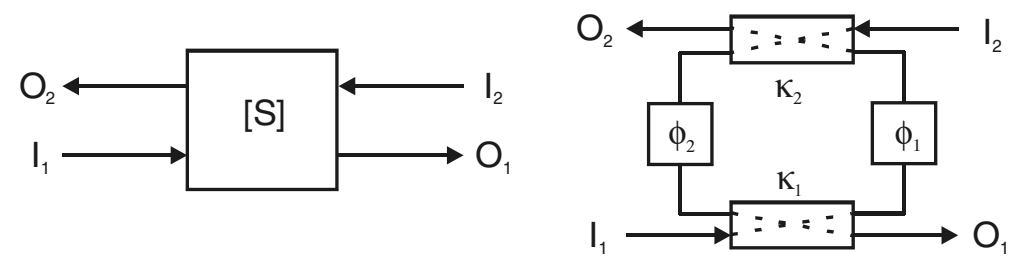

Fig. 8.4. Scattering matrix model of a microring resonator

This approach does not include polarisation effects, but it is sufficient in many cases for the derivation of fundamental design parameters for different material systems such as III-V semiconductors or silicon oxi-nitride (SiON) for example. We will present this approach in more detail in the following, as it illustrates relevant device characteristics and fundamental dependencies. As long as the MRs under consideration exhibit polarisation dependent characteristics without TE-TM mode conversion (cf. Sect. 8.2.) one may design MRs for polarisation diversity, while a significantly more sophisticated treatment is needed if TE- and TM-modes are no longer good eigenstates.

In the scattering matrix model the MR is modelled as two couplers, which couple a fraction $\kappa_{1}$ or $\kappa_{2}$ over to the cross port, and two delays $\phi_{1}$ and $\phi_{2}$ in-between as shown in Fig. 8.4. The optical fields in the inputs and outputs of the ring are related as follows:

$$
\left[\begin{array}{l}
O_{1} \\
O_{2}
\end{array}\right]=\left[\begin{array}{ll}
S_{11} & S_{12} \\
S_{21} & S_{22}
\end{array}\right]\left[\begin{array}{l}
I_{1} \\
I_{2}
\end{array}\right]
$$


with

$$
\begin{aligned}
& S_{11}=\frac{\mu_{1}-\mu_{2} \alpha_{r}^{2} e^{-j \Delta \omega}}{1-\mu_{1} \mu_{2} \alpha_{r}^{2} e^{-j \Delta \omega}} \\
& S_{21}=-S_{12}=\frac{\kappa_{1} \kappa_{2} \alpha_{r} e^{-j \Delta \omega / 2}}{1-\mu_{1} \mu_{2} \alpha_{r}^{2} e^{-j \Delta \omega}} \\
& S_{22}=\frac{\mu_{2}-\mu_{1} \alpha_{r}^{2} e^{-j \Delta \omega}}{1-\mu_{1} \mu_{2} \alpha_{r}^{2} e^{-j \Delta \omega}}
\end{aligned}
$$

where $\mu_{1,2}=\sqrt{1-\kappa_{1,2}^{2}}, \quad \alpha_{r}$ is the loss per roundtrip, $\Delta \omega=\omega_{0}-\omega$, with $\omega_{0}=\frac{c}{2 \pi R n_{g}} m$ being the resonance frequency, while $m$ is the resonance number. The delays $\phi_{1}$ and $\phi_{2}$ are related to the frequency and the optical path-lengths $L_{o p t l, 2}$ by $\phi_{1,2}=\frac{\omega L_{o p t l, 2}}{c}$, which are not necessarily identical to each other. The place of the couplers does not matter, as long as the total roundtrip phase is the same.

By use of this model one can extract values for the coupling constants from the desired functional behaviour and a given loss and radius. For example, in order to have as much power in the drop port as possible the two coupling constants must be the same [12]. In this case the device is said to be symmetric and has the lowest possible insertion loss in the drop port. Different parameters are found when optimizing the extinction ratio between the drop and through port. In that case the MR must be critically coupled [25], that means $\mu_{1}=\mu_{2} \alpha_{r}$. Under this condition all power is extracted from the through-port leading to the highest possible extinction ratio.

With the scattering matrix model, the influence of the loss parameter on the MR response can be determined as well. Parameters such as filtering bandwidth, insertion loss, crosstalk, and channel separation can be determined in this way. Three corresponding examples are given in Figs. 8.5 to 8.7 . 


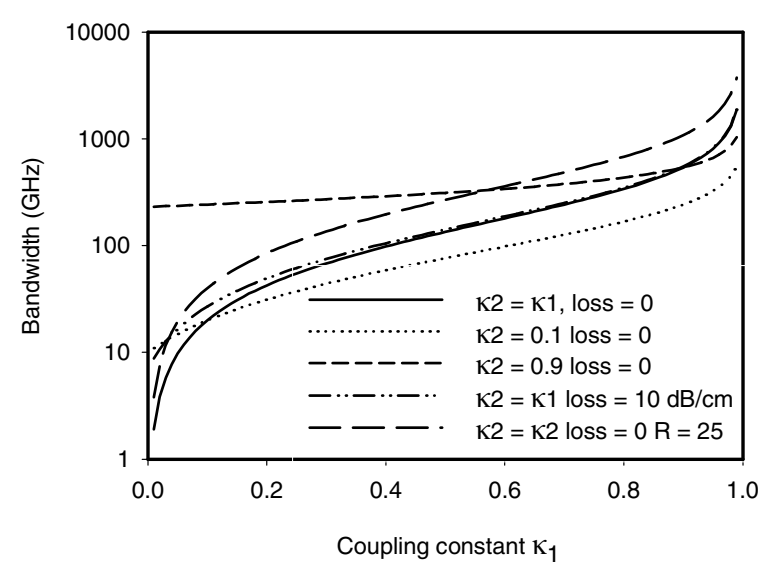

Fig. 8.5. Bandwidth of a MR as a function of coupling constant $\kappa_{1}$

Figure 8.5 shows the $3-\mathrm{dB}$ bandwidth of a microring based filter with $50 \mu \mathrm{m}$ radius, as given by (8.5), as a function of the coupling constant $\kappa_{1}$. The bandwidth increases as the coupling ratio gets higher. The effect of asymmetry in coupling is also shown in the figure by the lines with a fixed $\kappa_{2}$. Losses do not have a large impact on the bandwidth. Only for the lower coupling constants a difference can be seen. This figure focuses on bandwidth only, and one might conclude that having $\kappa_{2}$ fixed to 0.9 is favourable, since it assures high bandwidths over a wide range of $\kappa_{1}$. But when

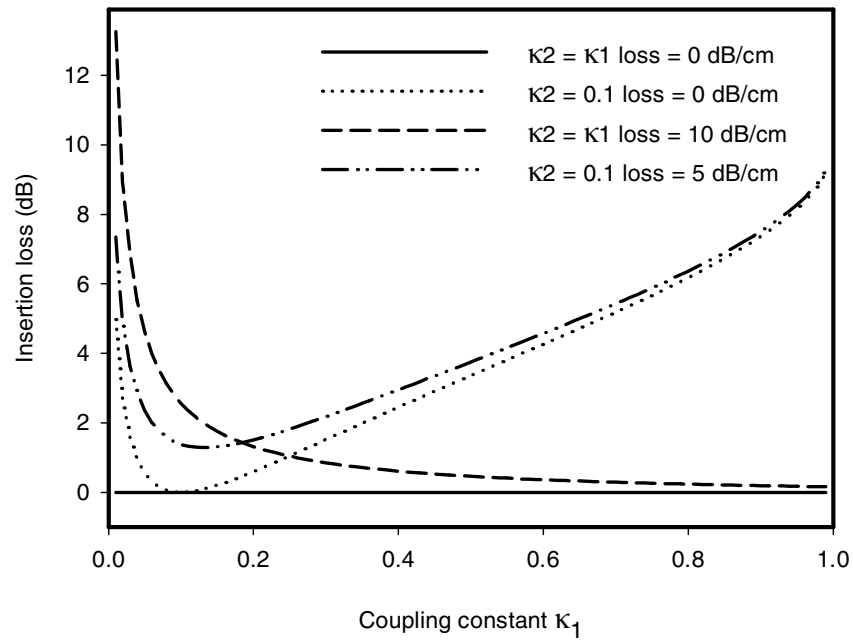

Fig. 8.6. Insertion loss for signals sent to the drop port as function of the coupling constant $\kappa_{1}$ for a MR on resonance 
looking at the insertion loss, which is another relevant parameter, this conclusion turns out to be premature as can be seen in Fig. 8.6.

Figure 8.6 shows the insertion loss in the drop port of a microring resonator as a function of the coupling constant $\kappa_{1}$ for the MR on resonance. The insertion loss (IL) can be calculated according to [11, 12]:

$$
I L=-\log \left(\frac{\kappa_{1} \kappa_{2} e^{-2 \pi R}}{\left(1-\sqrt{X_{1} X_{2}}\right)^{2}}\right)
$$

A symmetric lossless device has no insertion loss as can be seen from the solid line in Fig. 8.6. When the ring exhibits losses, the insertion loss increases, especially at lower coupling constants. The insertion loss does also increase with rising asymmetry of the coupling constants. Thus in order to get low insertion loss devices with bandwidths exceeding $10 \mathrm{GHz}$, the ring must be designed symmetrically with coupling constants above 0.4 .

In order to get maximum field inside the cavity, as required for example for all-optical switching, totally different specifications are derived. In this case the coupling constants must be in the order of 0.1 as is described in [26] and as can be seen in Fig. 8.7. The intra-cavity power $\left(\mathrm{P}_{\text {cav }}\right)$ has a maximum when the coupling constant is symmetric and relatively small (around 0.1).

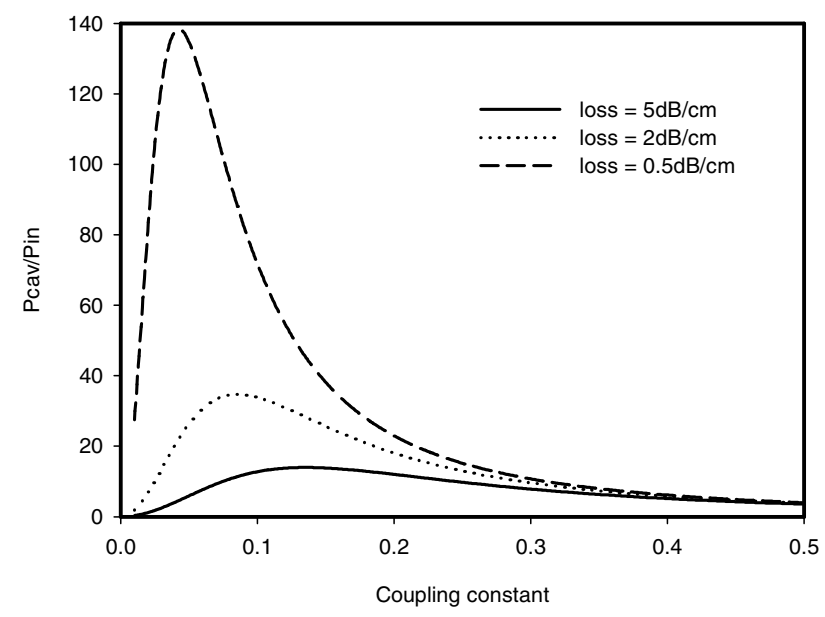

Fig. 8.7. Power inside cavity as a function of coupled fraction $\kappa$ assuming symmetric coupling for a ring with radius $R=50 \mu \mathrm{m}$ 
The scattering matrix model has proven to be a good tool to design single microring resonators, whatever the application-specific demands are. Furthermore, this model allows for extracting 'experimental' parameters by fitting the model to measured data, and the scattering matrix model is also applicable to more complex devices. In particular, cascading more than one ring can simply be expressed by matrix multiplication (cf. Sect. 8.6).

A design parameter of high relevance is the microring radius $\mathrm{R}$. Via the round trip path length the FSR (8.1) and the finesse (8.3) are radius dependent, and $\mathrm{R}$ affects the finesse by the round trip losses as well. For telecom applications in the $1.5 \mu \mathrm{m}$ wavelength range the FSR should be $>35 \mathrm{~nm}$ for the C-band for example. This implies very small rings with $R<6 \mu \mathrm{m}$, and this requires a high index contrast technology in order to avoid excessive bending losses. The trade-off between large FSR and low resonator loss can be circumvented by using more than one ring. When two rings of different radii are used, the Vernier effect [27-29] causes the total FSR to be a multiple of the respective single ring FSRs according to:

$$
F S R_{t o t}=N \cdot F S R_{1}=M \cdot F S R_{2}
$$

with $N, M$ being integers without common divisor (cf. Sect. 8.6.4).

\subsubsection{Geometry of Single Microrings}

Microrings can be designed and fabricated in two generic coupling configurations as shown in Fig. 8.8. When the ring and waveguide are structured in the same waveguiding layer the configuration is called 'laterally coupled'. When the ring and the waveguides are in different layers the configuration is called 'vertically coupled'. The vertical coupling configuration has the advantage that the coupling depends mainly on the thickness of the layer in between, which can be controlled very accurately during deposition, however at the expense of an additional processing step for the ring layer. The lateral configuration uses a single layer only, but requires very accurate lithography and etching processes to open the gap between the straight waveguide parts and the ring with high precision. Another advantage of the vertical configuration is that the ring and waveguide layers do not have to be the same thickness, which enhances the design freedom.

The fabrication of microring resonators is limited by the tolerances of the lithography and the etching processes. For the realization of the gap between the ring and the straight waveguide in a lateral configuration and using high index contrast, nanometre precision is required, which can only 
using high index contrast, nanometre precision is required, which can only be obtained by direct e-beam writing, focused ion beam milling or high precision wafersteppers. In the vertical configuration this problem is circumvented, as the coupling is now determined by the deposition process, where nanometre precision can be obtained more easily. In this case, however, alignment is an issue, as ring and port waveguides are structured in two separate lithographic steps. This is especially important in the case of symmetrically coupled devices.

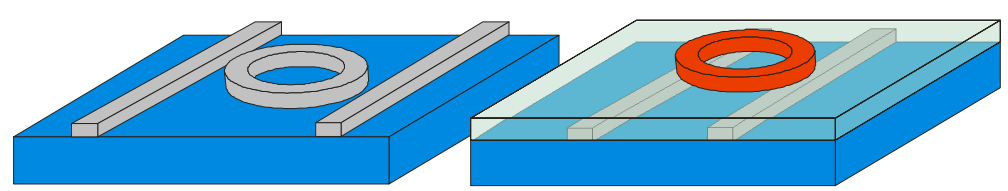

Fig. 8.8. 3D drawing of ring laterally coupled (left) and vertically coupled (right) to the straight waveguides

The optical modes of the straight waveguide cross-section and the bend cross-section can be calculated by the use of (bend-) mode solvers. Since the two modes are different in behaviour, they can not be calculated in a single step. In addition, different simulation tools may be needed. The most relevant parameters obtained by these calculations are i) the effective index, ii) the propagation and bending loss of the mode, and iii) the number of higher order modes besides the desired fundamental one. Figure 8.9 shows an example of a bend mode and straight waveguide mode calculated by a commercial mode solver [130]. The deformed mode profile with a shift to the outer rim of the curved waveguide (i.e. the right side in Fig. 8.9) is clearly visible.

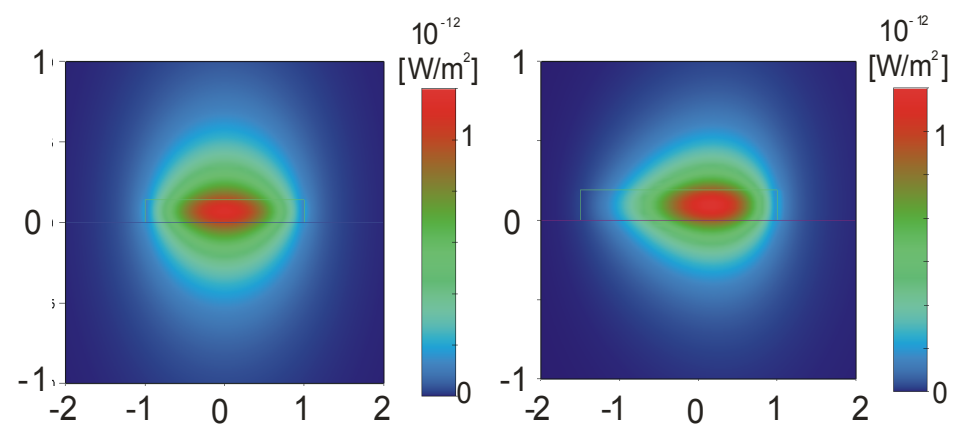

Fig. 8.9. Optical mode of straight waveguide (left) and bend (right) 
An important design consideration is the phase-matching between the mode that propagates within the ring resonator and the mode of the port waveguides. Ideally the effective indices of these modes should be the same. In general, however, this is not easily achieved because the index of the mode in the micro-resonator needs to be relatively high to keep the radiation-induced bending losses at an acceptable level. The effective index of the bend mode is therefore generally higher than the index of the straight waveguide, which is bound by the condition of mono-modality. Although the mono-modality condition is also important for the ring, a higher order bend mode has rapidly increasing losses.

The mismatch in phase causes the coupling between the port waveguides and the ring resonator to be less efficient as illustrated in Fig. 8.10, which shows calculations for a coupler, which consists of a straight waveguide vertically coupled to a bent waveguide as schematically given in the right part of the figure. The vertical distance between the two is $1 \mu \mathrm{m}$, the lateral distance is the gap. The calculation assumes waveguide indices of 1.97, an index of 1.45 for the surrounding, and a radius of $50 \mu \mathrm{m}$. According to Fig. 8.10 the maximum achievable coupling constant gets the lower the larger the phase mismatch. In the modelling described above losses inside the coupler region were not taken into account. However, these can be included by extending the scattering matrix as described e. g. in [31].

The dependence of bending losses on index contrast is illustrated in Fig. 8.11. Parameters for the calculation are $n_{\text {sep }}=n_{\text {buff }}=1.45$ and $n_{w g}=1.97$ (corresponding to a nitride waveguide). As can be seen the bending losses
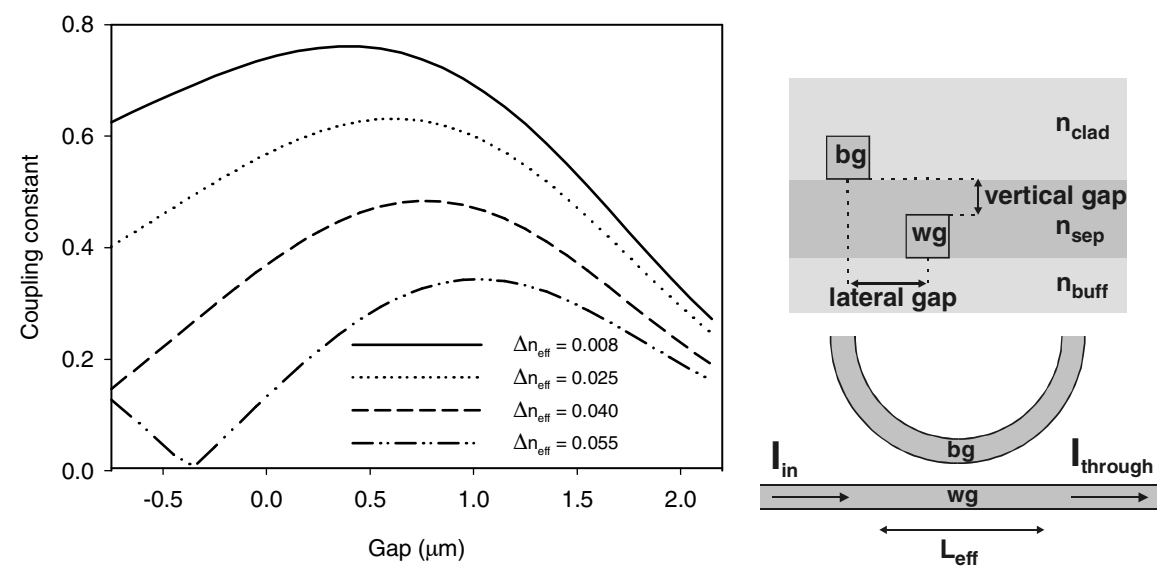

Fig. 8.10. Effect of phase-mismatch $\left(\Delta n_{\text {eff }}\right)$ on the coupling constants for various lateral gaps 


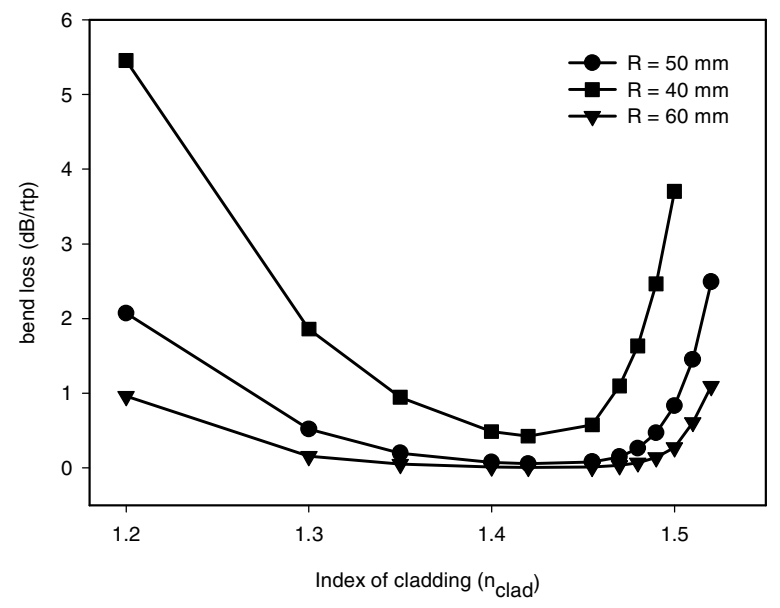

Fig. 8.11. Bending losses of SiON microrings of different radii for different cladding indices

have a minimum around $n_{\text {clad }}=1.45$. Furthermore, when the contrast decreases the bending losses increase. So higher index contrasts allow smaller radii, which implies larger FSR. However, when the index of the cladding layer is increased over that of the buffer and separation layers, the losses increase again, counter intuitively. But as explained in [32] the lateral contrast decreases in this case, which causes the losses to increase.

\subsubsection{Group Delay and Dispersion}

For applications in telecommunication the time behaviour of the microring or alternatively the frequency response to a time-varying signal is of great importance. Since the filter is a resonant filter, the delay depends on the frequency (wavelength) position with respect to the resonance. This dependence can conveniently be described by the structural or quadratic dispersion $D$ [9]. This dispersion is the second derivative of the transmission phase-response $\varphi(\omega)$ with respect to frequency. The normalized groupdelay $\tau_{n}$ is the negative derivative of the phase-response with $T$ being the inverse of the FSR:

$$
\begin{aligned}
\tau_{n} & =-\frac{d}{d \omega} \varphi(\omega) \\
D & =-c \frac{T}{\lambda^{2}} \frac{d}{d \omega} \tau_{g}(\omega)
\end{aligned}
$$



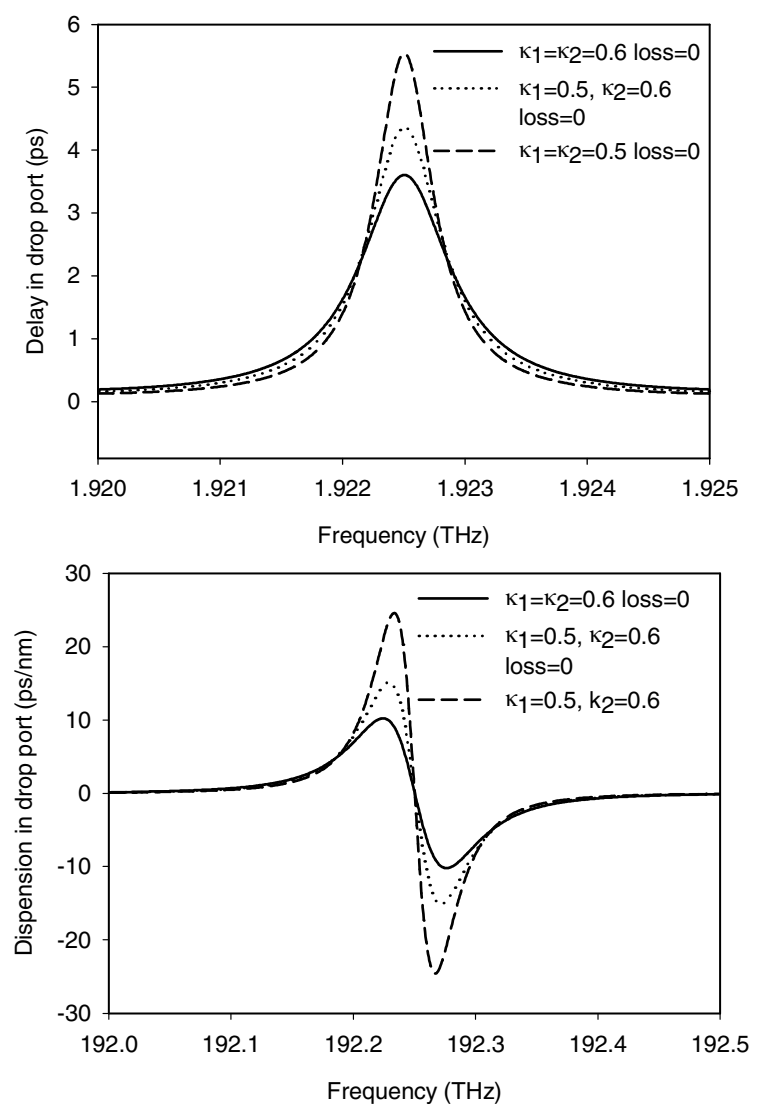

Fig. 8.12. Simulated absolute group delay (top) and dispersion (bottom) in microrings for various coupling constants

The absolute group delay is given by $\tau_{g}=T \tau_{n}$. Figure 8.12 shows a typical simulation of the group-delay and the dispersion response of the drop port of a single ring with radius $50 \mu \mathrm{m}$. In the symmetric case $\left(\kappa_{1}=\kappa_{2}\right)$ the through port exhibits the same response as the drop port.

According to Fig. 8.12 the absolute delay increases, when the coupling constants decrease (in that case $F$ and $Q$ increase), since more time is needed to 'build up' the signal. Also non-symmetric coupling constants increase the delay as can be seen comparing the dotted line with the solid one. This can be intuitively explained since raising one of the coupling constants, effectively decreases the finesse. At these higher coupling constants loss does not have much influence on the drop phase response. Larger ring radii cause higher delays, as it takes longer to get into resonance. 


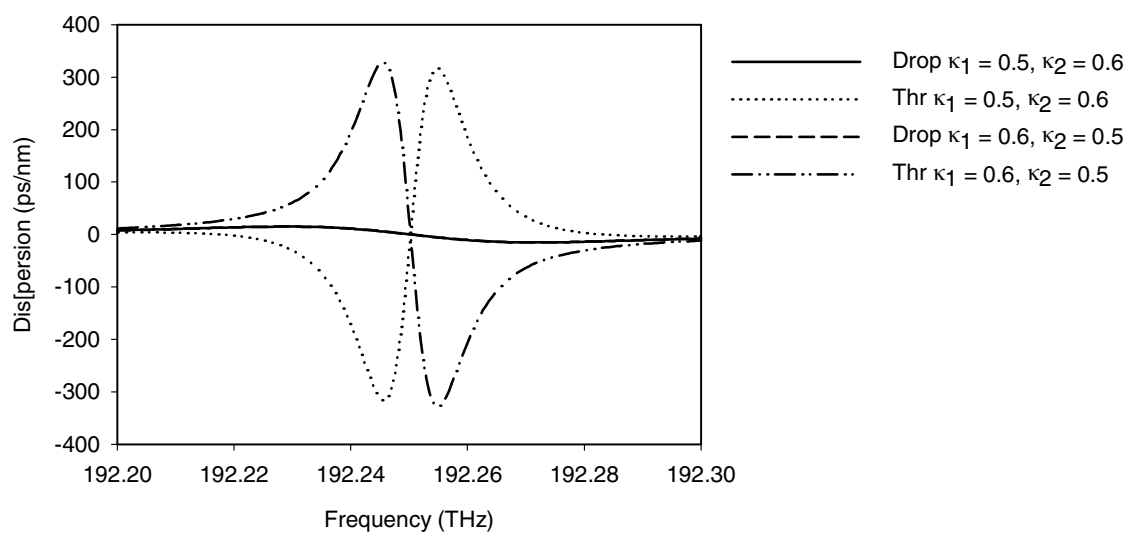

Fig. 8.13. Dispersion in drop and through port for two different kinds of asymmetry

Figure 8.12 also shows the simulated dispersion for various coupling constants and the effect of asymmetry. In the case of symmetric coupling $\left(\kappa_{1}=\kappa_{2}\right)$ the total dispersion experienced by the signal leaving the MR from the drop and the through port is moderate, and it is identical for both ports. However, in the case of asymmetric coupling $\left(\kappa_{1} \neq \kappa_{2}\right)$ the dispersion observed from the through port gets significantly larger (note the different scales of Figs. 8.12 and 8.13!) and the shape of the dispersion curve is inverted, if $\kappa_{1}$ and $\kappa_{2}$ are interchanged. This is illustrated in Fig. 8.13. The minimum phase filter becomes a maximum phase filter [9]. This effect can be used in filters used to optimize the time domain parameters of a signal i. e. dispersion compensators.

\subsubsection{Other Micro-resonator Geometries}

The shape of MRs is evidently not restricted to a circle. Nearly any geometrical path that provides optical feedback will act like the microring. A frequently used geometry is the racetrack [33] which is shown in Fig. 8.14. In this geometry the couplers of the resonator are straight waveguides that allow accurate control of the coupling constants at the expense of being somewhat larger and consequently having a reduced FSR.

Another common shape is the disk [34] instead of a ring as shown in Fig. 8.14. A disk is more difficult to make single mode, since the lateral width of the cross section is large. But since the lateral contrast of the disk is higher than the contrast for a ring, potentially lower losses can be obtained. 

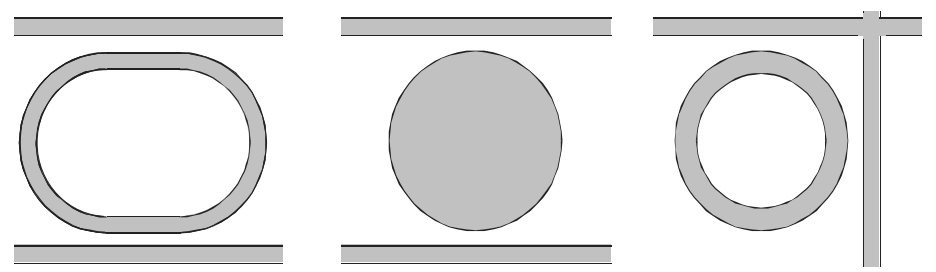

Fig. 8.14. Other microring resonator shapes: Racetrack (left), disk (middle) and Manhattan configuration (right)

The place of the adjacent waveguides is not important. As can be seen from (8.8) only the total roundtrip length influences the response, but not the place of the coupling regions. An additional geometry is the so-called Manhattan configuration as shown in Fig. 8.14. This structure allows for optimum use of area and is scalable to a large extent.

\subsection{Tuning and Modulation of Microrings}

Until now the ring was assumed to be totally passive, i. e. all geometrical and materials parameters being constant in time. To add functionality or to overcome fabrication errors the MR can be made active by varying (tuning) some of the parameters and consequently its wavelength response. Furthermore, additional functionality can be added by externally tuning the roundtrip loss or coupling constants of the ring.

\subsubsection{Resonance Wavelength Tuning}

The resonant wavelength of a MR can be tuned in several ways. The most straightforward approach is to change the effective index of the ring by any of the following means:

- Thermo-optic effect: by applying heat to the ring the refractive index of the material changes [35]

- Electro-optic effect: an electrical field causes the change in refractive index [36]

- Carrier injection: optical pumping creates free carriers (single photonor two-photon absorption), which change the loss parameter and the refractive index of the material $[37,38]$

- Changing the material (sensor) $[39,40]$

- Opto-optical effect: the light itself causes a change in index via nonlinear effects [11] 


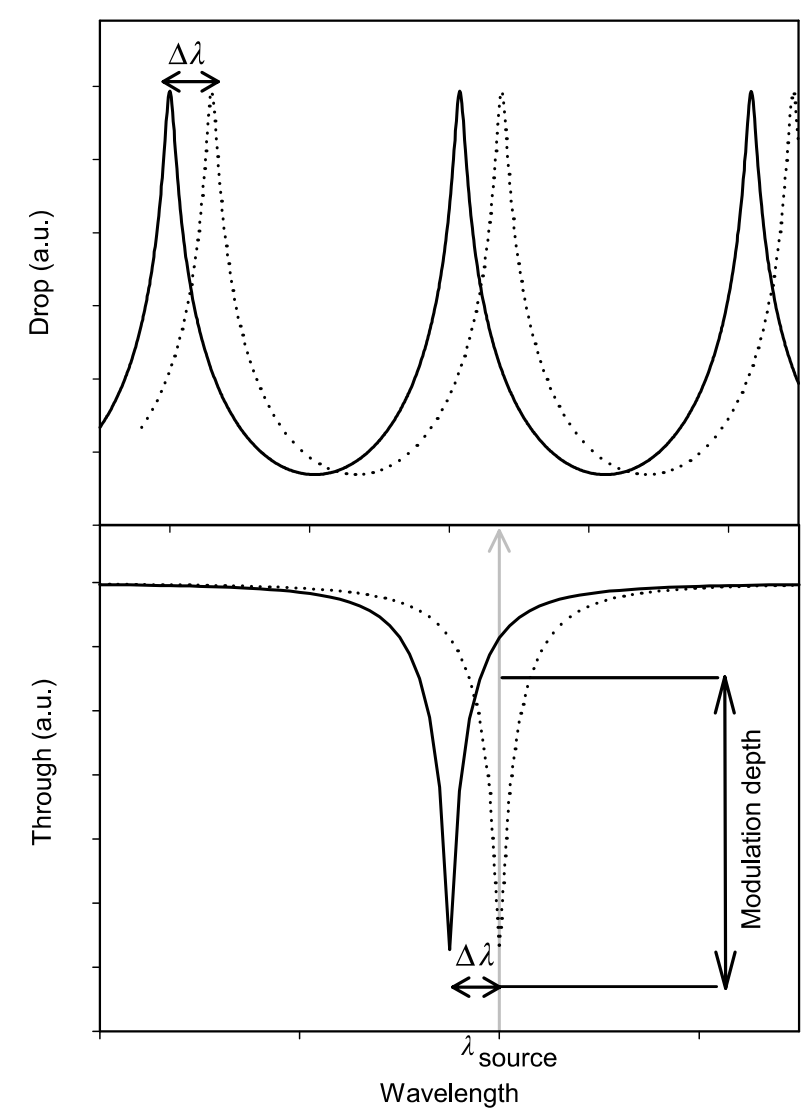

Fig. 8.15. Tuning the passband of a MR filter to different wavelengths (top) and modulation by tuning the MR wavelength response for fixed operation wavelength (bottom)

A change in effective index shifts the total wavelength response by an amount of $\Delta \lambda$. This shift can be used in filter applications to tune the passband to the a desired wavelength. The principle of tuning is shown in Fig. 8.15.

The same phenomenon leads to a different functionality, when the operation wavelength $\lambda_{\text {source }}$ is kept constant. A small change in effective index around the steep slope of the MR response induces a modulation at $\lambda_{\text {source }}$ with high extinction ratio.

The thermo-optic effect can be used by applying a thin film heater on top of the ring. The heater must be placed as close as possible to the ring to minimize the driving power. On the other hand, the heater can not be placed too close, since the metal from the heater will induce large additional losses inside the ring. 


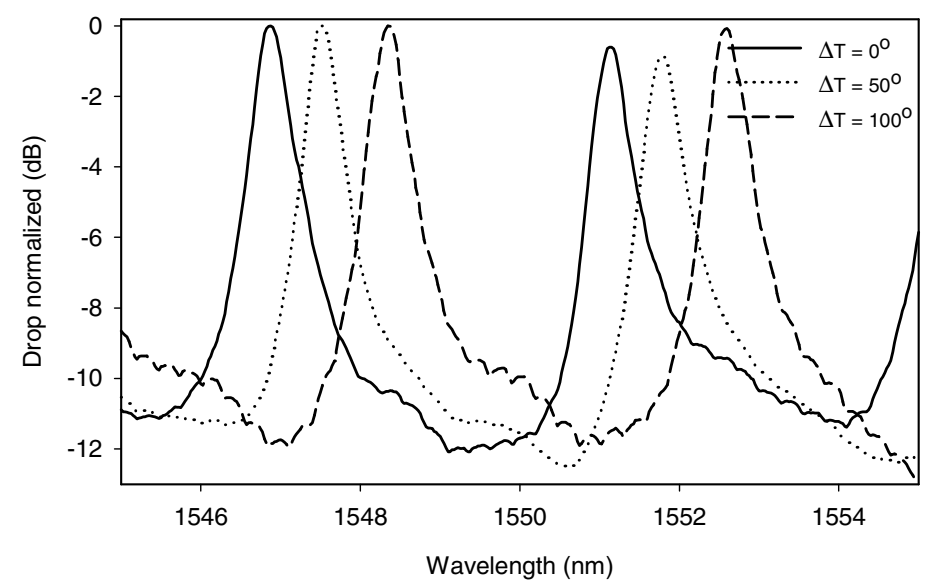

Fig. 8.16. Shift of drop response as function of heater temperature of a $\mathrm{SiO}_{2} / \mathrm{Si}_{3} \mathrm{~N}_{4} \mathrm{MR}$ with radius $50 \mu \mathrm{m}$

The shift in centre wavelength of the ring $\Delta \lambda_{\mathrm{c}}$ is a function of the difference in effective index induced by heating the device, according to:

$$
\Delta \lambda_{c}=\frac{\lambda \Delta n_{\text {eff }}}{n_{\text {eff }}}
$$

The change in effective refractive index induced by the heater depends largely on the materials used and the distance between the heater and the ring. Glass like materials have a temperature-dependence of the refractive index $(\mathrm{d} n / \mathrm{d} t)$ of around $10^{-5}$, whereas polymers have a coefficient being an order of magnitude higher. Thermal shifts of $20 \mathrm{pm} / \mathrm{mW}$ have been shown for glass-based MRs [41], see Fig. 8.16. For polymer MRs this shift is about $1 \mathrm{~nm} / \mathrm{mW}$ [42], i. e. around two orders of magnitudes higher, which can be explained by the larger thermo-optic coefficient and a better conductance of the heat as described in [43]. In conclusion, thermal tuning is simple to implement, but the thermo-optic effect is slow with typical response times in the order of hundreds of microseconds. This can be sufficient for switching applications, but other effects must be used for modulation applications.

The electro-optic effect changes the $i$-th component of the refractive index $n_{i}$ as function of the applied electric field $E_{k}$ according to:

$$
\Delta\left(\frac{1}{n_{i}^{2}}\right)=\sum_{k} r_{i k} E_{k}
$$


With $r_{i k}$ the eo tensor $[44,45]$. The direction of change of refractive index is determined by the material and can be positive as well as negative. Applying a reverse voltage will lead to the reversal of the effect. The electro-optic effect is inherently much faster than the thermo-optic effect. In practice now the limiting factor will be the 'build-up' time of the resonance given by

$$
\tau_{c a v}=\frac{F \cdot R \cdot n_{g}}{c}
$$

For well designed ring resonators electro-optic modulation speeds of several tens of $\mathrm{GHz}$ can be expected. In recent experiments [45] modulation up to $1 \mathrm{GHz}$ has been demonstrated in a Mach-Zehnder interferometer loaded on one arm with a microring. The device has been fabricated in PMMADR1 polymer, the relevant electro-optic coefficient is $r_{33}=10 \mathrm{pm} / \mathrm{V}$ $[44,45]$.

In semiconductor materials, with highly confining waveguides, which have a bandgap energy higher than the photon energy, it is possible to change the refractive index by injecting free carriers [46]. An empirical relation between the change in refractive index and the carrier density [47] leads to an index change of around -0.002 .

For all-optical or opto-optical tuning mostly the Kerr non-linearity of a material is used [11] i.e. the total effective refractive index of the material is dependent on the intensity of the light $\left(I_{\text {eff }}\right)$ :

$$
n_{\text {eff }}=n_{\text {eff }, 0}+n_{\text {eff }, 2} I_{\text {eff }}
$$

where $n_{e f f, 2}$ is a parameter dependent on the Kerr non-linearity. Recently, $\mathrm{GHz}$ modulation in silicon-on-insulator (SOI) MRs based on this effect has been shown [38].

\subsubsection{Resonance Wavelength Trimming}

Statistical errors in the radius during fabrication can be cancelled by post deposition trimming. In the methods described above an active function is introduced to change the resonant wavelength. When permanent changes are needed in order to compensate for fabrication errors, other methods are required. The effective index of the ring can e. g. be altered by locally injecting high optical power by a laser. This post-fabrication process can be used to trim every MR to the proper position [48]. For low-cost mass production, however, the need of trimming should be completely avoided. 


\subsubsection{Loss Parameter Tuning}

In the previous section methods of tuning were discussed, where the optical roundtrip path length was changed, which resulted in a shift of the resonant wavelength. Alternatively, the roundtrip loss can be changed, which affects the Q-factor and the shape of the resonance curve. In this way a MR can be used as space-switch: at the resonance in high Q, weakly coupled MRs practically all power is directed to the drop port, but at low Q to the through port. There are several methods of controlling the $\mathrm{Q}$ of the cavity dependent on the materials in the system. In semiconductor devices electro-absorption or free carrier injection can be used to control the loss parameter [46]. Other materials like polymers and glasses allow for doping with rare-earth ions like Erbium [49]. In these materials the losses can be largely reduced by optical pumping. In this way switching of light by light is feasible, i. e. opto-optical switching.

\subsubsection{Coupling Constant Tuning}

Finally, the individual coupling constants can also be tuned. This has been done mainly in relatively large racetrack structures, where enough coupling length is present to allow for thermo-optic tuning restricted to the coupling sections [50]. Furthermore, some more exotic methods have been demonstrated making use of MEMS (Micro Electro-Mechanical Systems) technology. Fleming et al. changed the coupling between port waveguides and ring by moving the ring, which was attached to a membrane, in a controlled way by electro-static forces to the evanescent field of the waveguides underneath. In this way light at the resonance wavelength is coupled from one waveguide to the other [51].

\subsection{Characterisation Methods}

The full characterisation of microring based devices requires the determination of their amplitude and phase characteristics (as outlined in detail in Chap. 2). Wavelength-dependent measurements of transmission, add, and drop characteristics and of crosstalk properties as well can be done using broadband or tuneable narrow band light sources. Essential points have been outlined in Chap. 4, Sect. 4.7 for the characterisation of AWGs and hold for microrings as well. Particular requirements for the investigation of high index-contrast devices are sub- $\mu \mathrm{m}$ precision with negligible drift over tens of minutes. 


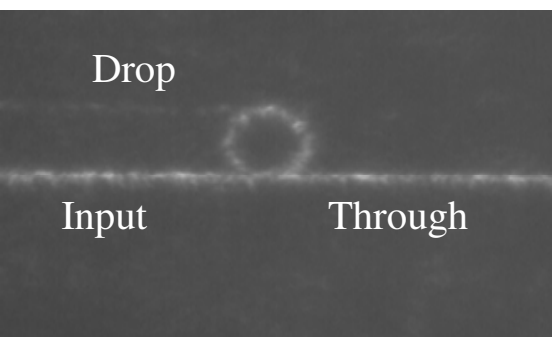

(a)

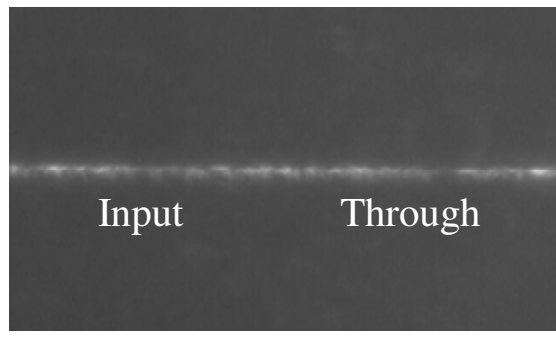

(b)

Fig. 8.17. IR Camera image of MR on-resonance (left) and off-resonance (right)

Another characterisation method is a lens system and an IR camera placed on top of the device under test. In this way scattered light can be captured, and by image processing also wavelength scans of particular parts of the image can be made [52]. The technique provides the possibility to measure every point on the device, and this is particularly useful in more complex systems, where not all inputs and outputs of the individual rings are directly connected to chip input/output ports. With the camera technique one can look 'in between' rings, and on-chip insertion loss can be assessed rather easily, as scattered intensity inside the waveguides decreases along the propagation direction. The principle is limited to devices or channels that exhibit sufficient scattering, thus it is primarily suited for device characterisation in an early development stage. Figure 8.17 shows an example of a $\mathrm{SiO}_{2} / \mathrm{Si}_{3} \mathrm{~N}_{4} \mathrm{MR}$ with a radius of $25 \mu \mathrm{m}$ on resonance (left picture) and off resonance captured by an IR camera setup.

The phase characteristics of microring-based devices can be measured by techniques such as the phase-shift method $[53,54]$ or by optical lowcoherence reflectometry (OLCR), which are discussed in detail in Chap. 2, Sects. 2.3.1 and 2.3.2.

OLCR measurements can be performed in reflection and in transmission as well. The distance between consecutive interference peaks is a measure for the FSR, while the exponential envelope of the interference peaks e. g. provides information on the coupling constants and the losses $[17,50,55]$.

\subsection{Multiple Ring Resonator Devices}

For many applications, in particular in fibre optic communication, the characteristics of single microrings are not well suited. However, much more favourable device performance can be achieved, if a number of microrings 
are combined, and this can be done in a variety of architectures. This will be outlined in more detail in the following section.

\subsubsection{Higher Order Filters}

Higher order MR filters are devices, where several feedback paths contribute to the response of the filter. Figure 8.18 shows two different setups, normally designated as serial cascade $[9,56-60]$ and parallel cascade $[52,61-66]$ in the literature. In both cases, the additional feedback paths result in an improved filter response, i.e. a more flat-top response in the pass-band and a steeper roll-off at the pass-band edges. The design of a serial cascade using the z-transform approach is described in detail e. g. in [9].

Concerning the parallel cascade a number of different design concepts have been reported in the literature. Examples are considering the cascade as a generalized DFB grating $[63,66]$, describing the filter in terms of a prototype filter as known in the microwave domain [61] or using the scattering matrix description $[64,65]$. Recently, the z-transform description of these filters has also been applied [67].

In the serially cascaded structure it is necessary to control the resonance wavelength of each ring in the filter accurately. In the parallel cascade the distance between the rings must be well controlled with sub-wavelength precision. In both cases the filter responses can be further improved by special designs of all the coupling constants in the cascade [66]. By apodizing the coupling constant for each individual ring along the array, even better filter shapes can be obtained.

Higher order filters is a serial cascade of 3 to 11 rings have been made by Little Optics in their Hydex ${ }^{\mathrm{TM}}$ technology, which enables the fabrication of lightwave circuits including microring structures with appealing characteristics. Hydex ${ }^{\mathrm{TM}}$ is claimed to overcome all the limitations of other high$\Delta n$ systems, the details of which are not disclosed so far. However, it is deposited through a conventional chemical vapour deposition process, its

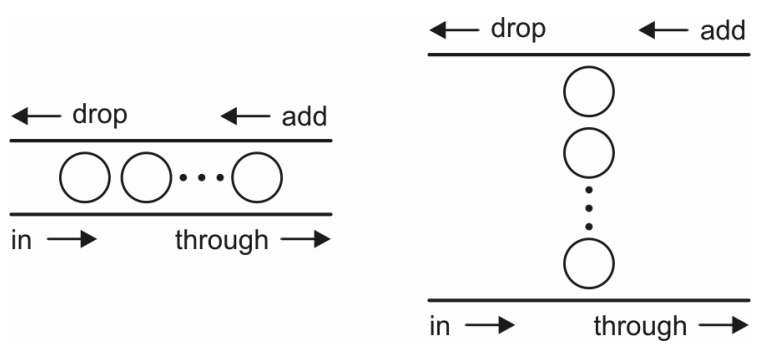

Fig. 8.18. Serially (left) and parallel cascaded ring filters (right) 
refractive index contrast is adjustable from $0 \%$ to over $20 \%$, the fabrication does not require any annealing step, and the material absorption is low all over the S-, C-, and L band [68].

Figure 8.19 shows a serial cascade of three rings and the transmission spectra of $1^{\text {st }}-, 3^{\text {rd }}$-, and $11^{\text {th }}$-order serial filters.
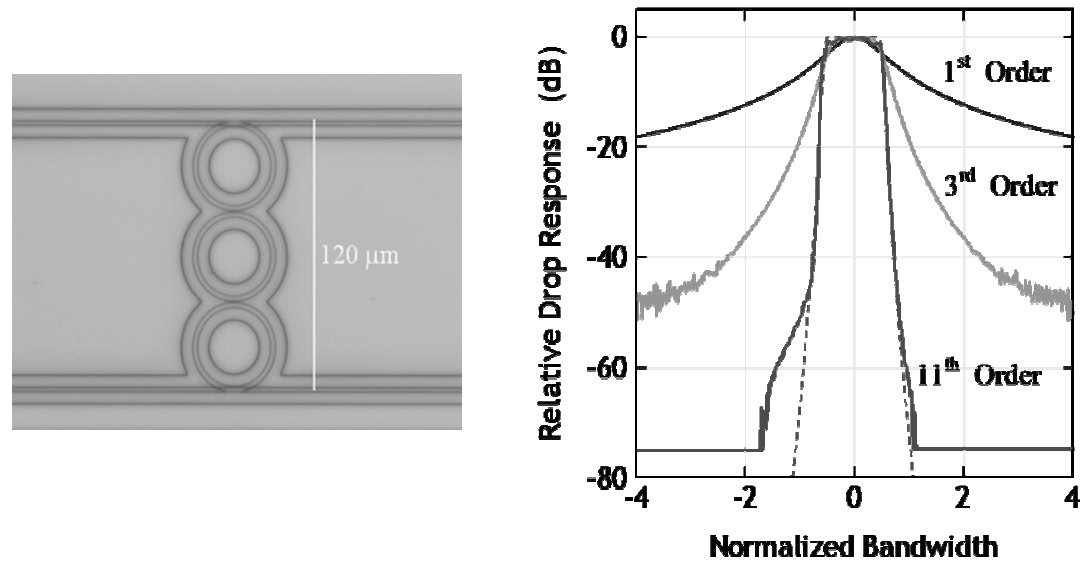

Fig. 8.19. (a) $3^{\text {rd }}$ order filter and (b) Transmission spectra of different order ring filters (Little Optics Inc.)

As can be seen, the roll-off of the filter characteristics can be freely adjusted by choosing an appropriate number of cascaded rings. Typical characteristics of these commercially available tuneable filters based on cascaded rings are compiled in Tab. 8.1.

Tab 8.1. Characteristics of tuneable ring filters (Little Optics Inc.)

\begin{tabular}{lcccl}
\hline Channel spacing & 25 & 50 & 100 & $\mathrm{GHz}$ \\
\hline Tuning range & & $192.1-196.1$ & & $\mathrm{THz}$ \\
3-dB pass-band & $>17$ & $>35$ & $>42$ & $\mathrm{GHz}$ \\
Insertion loss & $<5$ & & $\mathrm{~dB}$ \\
PDL & $<0.5$ & $\mathrm{~dB}$ \\
\hline
\end{tabular}

\subsubsection{Wavelength-selective MR Switch}

A schematic drawing of a MR based switch is shown in Fig. 8.20. This structure is based on two MRs connected through a straight waveguide and acts as a wavelength selective switch. The first MR selects a certain wavelength. 

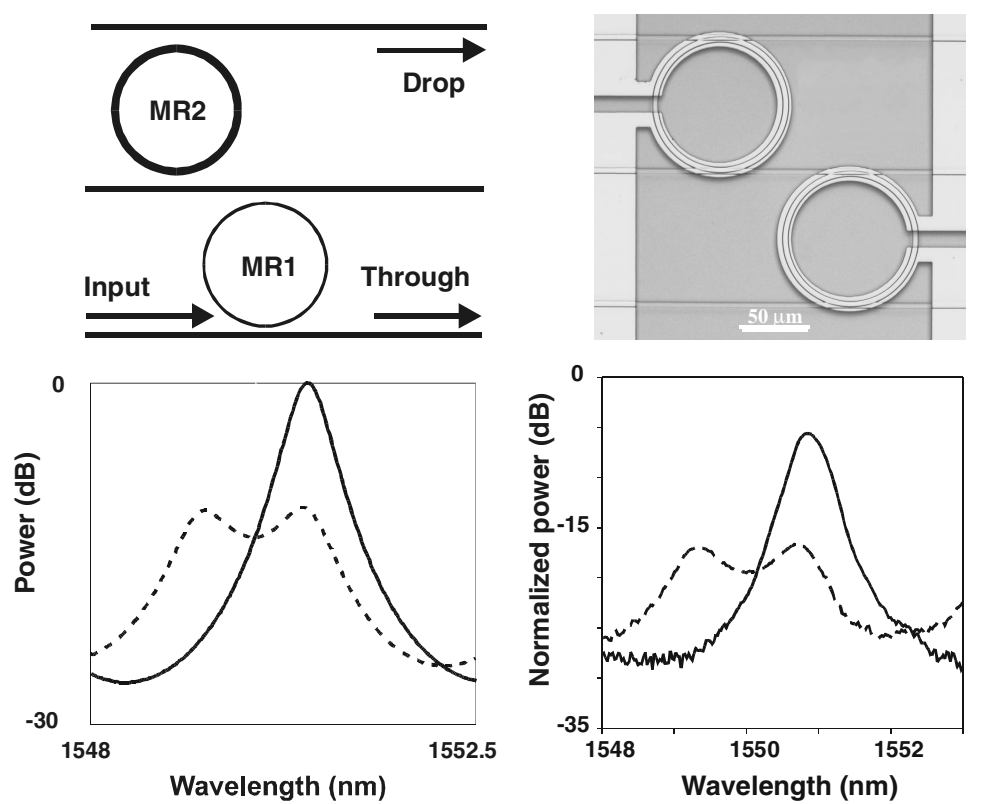

Fig. 8.20. Wavelength selective switch based on two MRs: schematic lay-out and microscope picture of device (top) and wavelength response of the drop port (bottom). Lower left: simulated response of single ring; solid line: switch in ON-state, dotted line: switch in OFF-state. Lower right: experimental results

The second ring, which is tuneable, thereafter switches this wavelength to the drop port or to a non-used channel leading to an absorber [54].

\subsubsection{Reconfigurable Optical Add/drop Multiplexer}

Another example of complex structures based on MRs is the $N \times M$ matrix switch based on the Manhattan configuration [69] as shown schematically in Fig. 8.21. This compact configuration allows adding and dropping any of the $M$ wavelengths to any of the $N$ input/output ports. In order to improve the performance each building block consisting of a single MR can be replaced by a higher order filter $[69,70]$.

The SiON technology looks promising as a platform for these complex MR structures. With a large index range between $1.45\left(\mathrm{SiO}_{2}\right)$ and $2\left(\mathrm{Si}_{3} \mathrm{~N}_{4}\right)$ $\mathrm{SiON}$ allows for relatively high index contrast, low-loss devices with small bending radius $[19,71]$. This is a prerequisite for complex structures, since multi MR devices with large FSR can only meet the specifications when they are small in size and have low losses. It is therefore not surprising that quite some interest has been shown for MRs in the SiON technology, see 


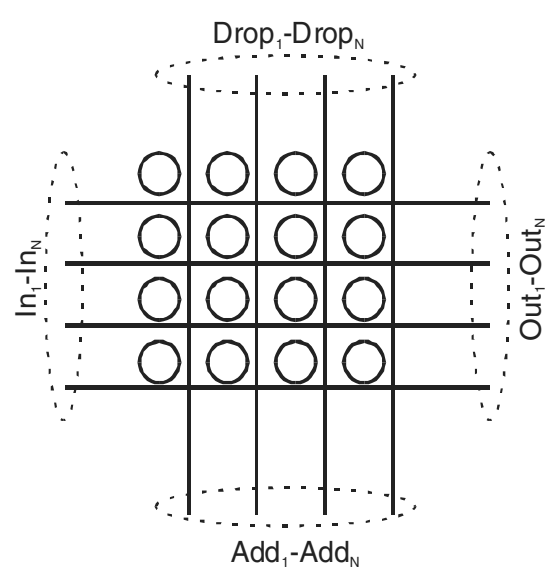

Fig. 8.21. $N \times M$ Manhattan microring configuration with $N$ input/output channels and add/drop ports for each of the $M$ wavelengths

for example $[20,62,69,72]$. Some of the previous figures in this chapter (Fig. 8.1, Fig. 8.17 and Fig. 8.20) are MR structures in SiON technology as well. As another example in Fig. 8.22 a single row of a cross-grid structure already discussed above is shown. This structure, known as a Reconfigurable Optical Add/Drop Multiplexer (ROADM) is based on $\mathrm{Si}_{3} \mathrm{~N}_{4}$ channels embedded in $\mathrm{SiO}_{2}$. The individual rings are thermally tuneable across the full FSR by means of a chromium thin film heater. In this way the ROADM can, for example, be switched performing as a 4 channel demultiplexer, where every ring filters a particular wavelength from the in-port to the respective drop-ports. Alternatively, when the microrings are tuned to the same wavelength, the structure can be used as a single wavelength broadcasting device, directing one input wavelength to more than one drop port. This is shown in the upper right picture in Fig. 8.22, were the through port of the device is shown when the four rings are set to different wavelengths (4 single channels) or all at the same wavelength (single combined channel). The lower right picture displays the response for the four individual add ports, when light enters one of the add ports and is directed to the through port. This demonstrates that every channel can add power to the through port.

The device was pigtailed and wire-bonded and can be controlled by a computer. It has an inter-channel crosstalk of $12 \mathrm{~dB}$, can tune to any wavelength within the FSR of $4.2 \mathrm{~nm}$ at a maximum driving power of $380 \mathrm{~mW}$ per channel. The device shows $12 \mathrm{~dB}$ extinction per channel in the through port and $17 \mathrm{~dB}$ in the drop port [73], and it has been tested at $40 \mathrm{Gbit} / \mathrm{s}$ without showing reduction of performance [74]. 

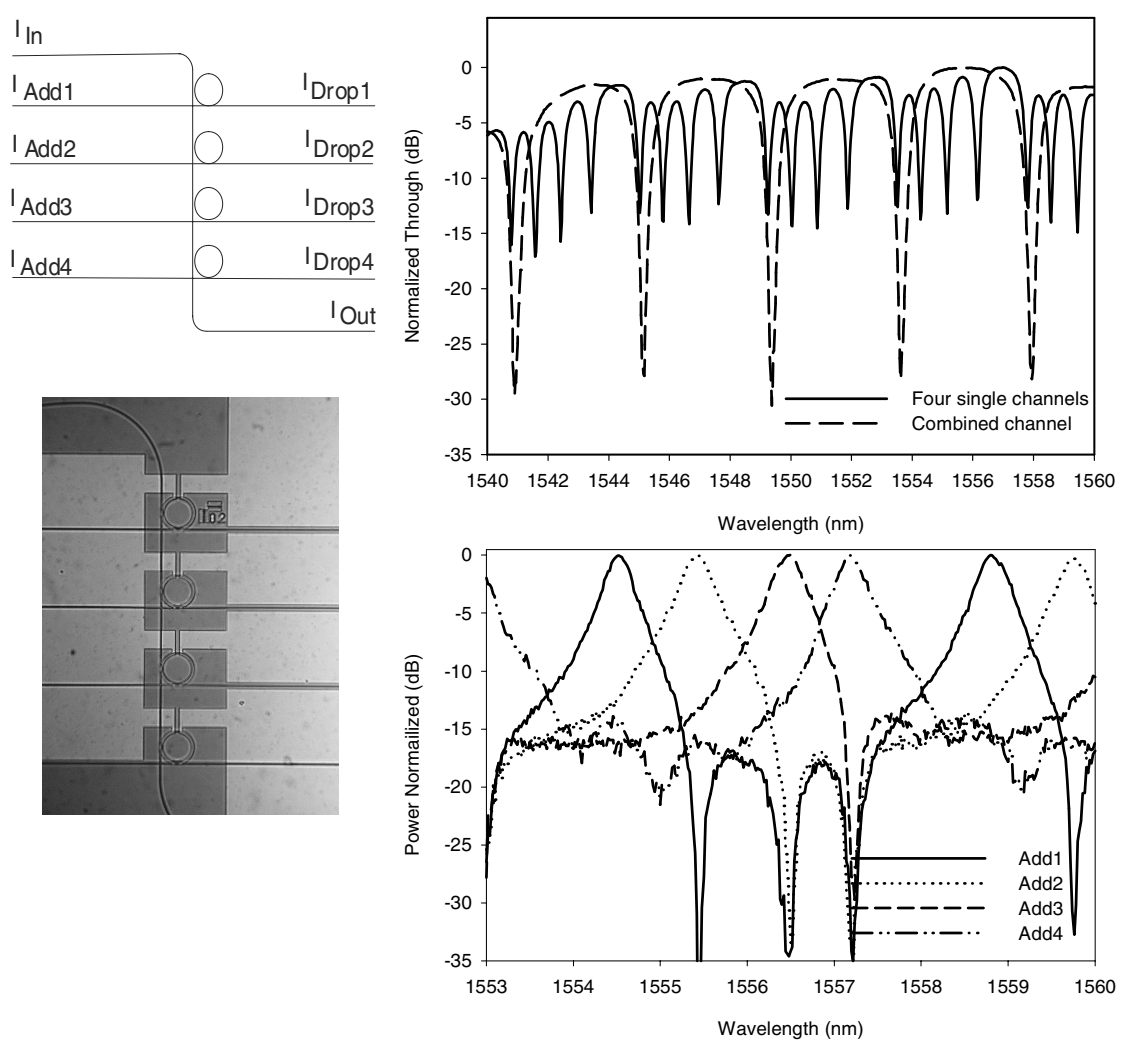

Fig. 8.22. Schematic (top left) and photograph (bottom left) of a $\mathrm{SiO}_{2} / \mathrm{Si}_{3} \mathrm{~N}_{4}$ MR based ROADM and the measured through (top right) and add (bottom right) response

Currently, there are several commercial suppliers of MR based products in SiON technology. Two of them Little Optics (recently acquired by Nomadics [68]) and Lambda Crossing [75] deliver products based on MR technology, whereas another one, LioniX [71] offers foundry services and MR technology for use by others.

\subsubsection{Microring-based Filters with Extended FSR}

For many applications in fibre optic systems the FSR of filters should be comparable to the width of the C- or the L-band, for example, i. e. several $10 \mathrm{~nm}$. According to (8.1) large FSR values require small bend radii (where the actual numbers vary widely depending to the material system chosen), and achieving several $10 \mathrm{~nm}$ FSR is demanding due to technological restrictions. 


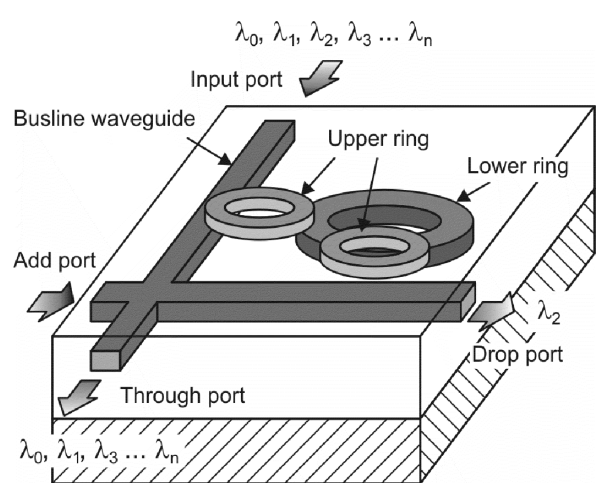

Fig. 8.23. Triply-coupled microring resonator filter in stacked configuration (after [77])

One means to increase the FSR without resorting to correspondingly small bend radii relies on the Vernier effect, i.e. microrings of different radius are combined, and the resulting device exhibits resonances only, where the individual resonances of all microrings involved match. (The approach is similar to one concept to design widely tuneable lasers by using gratings with slightly different pitch, which provide super modes with rather large wavelength separation [76], cf. also (8.10)). A corresponding microring-based device is illustrated in Fig. 8.23 [77].

The device has been fabricated in $\mathrm{SiO}_{2} / \mathrm{Si}$ and the ring radii are $28.5 \mu \mathrm{m}$ for the two smaller (upper) rings and $39.3 \mu \mathrm{m}$ for the larger (lower) ring corresponding to a FSR of the individual rings of 8 and $6 \mathrm{~nm}$, respectively, while the integrated filter exhibited about $20 \mathrm{~nm}$ FSR.

\subsubsection{Microring-based Dispersion Compensators}

Dispersion compensation is a functionality particularly needed in high-bit rate optical communication systems, since the adverse effects of dispersion increase proportional to the square of the data rate. Dispersion compensation can be accomplished by many approaches, including bulk optics and in particular fibre Bragg gratings (cf. Chap. 5, Sect. 5.5), but planar waveguide technology as well. Examples of the latter are finite impulse response filters implemented as resonant couplers [78] or infinite impulse response (IIR) filters, which exhibit periodic frequency characteristics and are thus particularly suited for dispersion compensation of multiple wavelength channels. Dispersion compensators using microrings rely on the all pass filter characteristics of MR-based two-port structures, and one example of a corresponding dispersion compensating filter is illustrated in Fig. $8.24[79,80]$. 

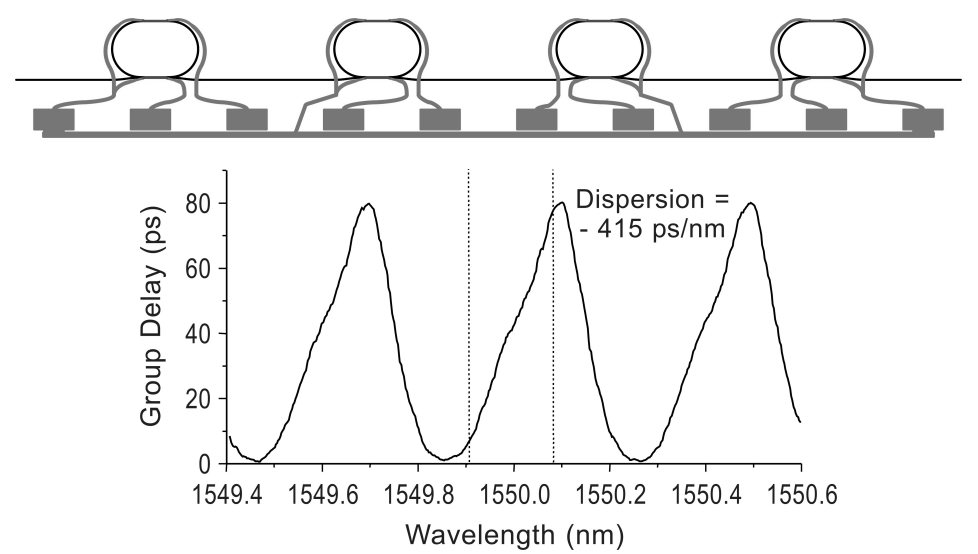

Fig. 8.24. Ring-resonator-based dispersion compensator. Upper part: device structure, lower part: device characteristics, after [80]

The tuneable dispersion compensator illustrated in Fig. 8.24 consists of four cascaded resonator loops and has been fabricated in high-refractiveindex contrast SiON technology. An absolute index contrast of 0.05 or $\Delta=3.3 \%$ between waveguide core and cladding allows minimum bend radii of $0.8 \mathrm{~mm}$ with barely measurable radiation loss and $0.55 \mathrm{~mm}$ radii with $<0.1 \mathrm{~dB}$ loss per $90^{\circ}$ bend [80], and small ring radii are required in order to get large FSR values. The device illustrated in Fig. 8.24 is made adjustable by the implementation of two heaters per microring, where one heater is used to vary the optical path length and the other to vary the strength of the coupler. In this way tuneable filters with linear delay slopes of $+100,+50,-50$, and $-100 \mathrm{ps} / \mathrm{nm}$ were generated, but filters with quadratic dispersion-slope values of +800 and $-800 \mathrm{ps} / \mathrm{nm}^{2}$ as well. The technology lends itself to mass production and is expected to find applications in fibre optic systems operating at 10,40 , or even higher data rates.

\subsection{Semiconductor-based Microrings}

Microring- (including microdisk-) based devices have been fabricated in GaAs/AlGaAs [81], and in the GaInAsP/InP material system [82-85] as well. MRs in III-V semiconductors (SC) are considered attractive, because they can be monolithically integrated with other (active) functionalities. They can be used as channel dropping filters, WDM demultiplexers, as notch filters, for optical switching [86], or for all-optical wavelength conversion $[81,87]$. 
Microrings and microdisks plus their straight coupling waveguides (WG) have on the one hand been fabricated as planar structures [81, 84], but in vertical coupler geometry as well [85]. The latter design is particularly favourable for the control of the coupling between the straight waveguides and the ring, however, the fabrication process is significantly more demanding (requires wafer bonding for example). The design of SC based microrings has mostly been based on ridge waveguides (RWs) and to a much lower extent on buried waveguide structures [88], which are considerably more difficult to realize.

Layer composition, thicknesses, and WG geometry are determined on the basis of various, partly conflicting requirements: the straight input/output WGs should enable efficient and alignment-tolerant coupling to optical fibres, coupling between the straight and the bent WGs should be efficient, guiding in the bent WGs (i.e. in the MR) should be strong enough to enable small bend radii. The geometry of the bent WGs may either be symmetric or asymmetric [82,84], where a steeper etch at the outside wall of the RW-based MR structure assures a particularly large difference of the effective refractive indices and consequently the strong confinement of the guided mode, which is required for low radiation losses in small bend radius microrings. An asymmetric, deeply etched bent WG is illustrated in Fig. 8.25.

As discussed in Sect. 8.2.3 microrings tend to be polarisation dependent, and this is particularly true for RW-based semiconductor microrings. In applications, where the state of polarisation of a lightwave entering a microring is random, polarisation diversity could solve the problem, although at the expense of extra complexity. On the other hand, if the polarisation needs alignment anyway, for example in all-optical wavelength conversion, the polarisation dependence of the microring characteristics is less of an issue.

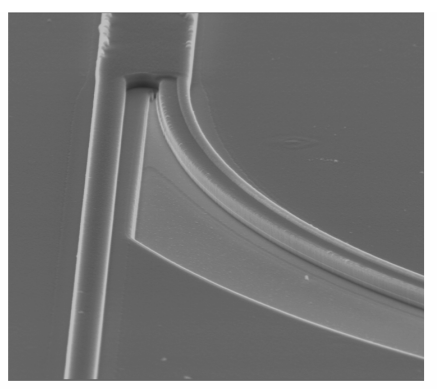

Fig. 8.25. SEM picture of bent WG region illustrating deep etch at the outside microring wall, after [84] 


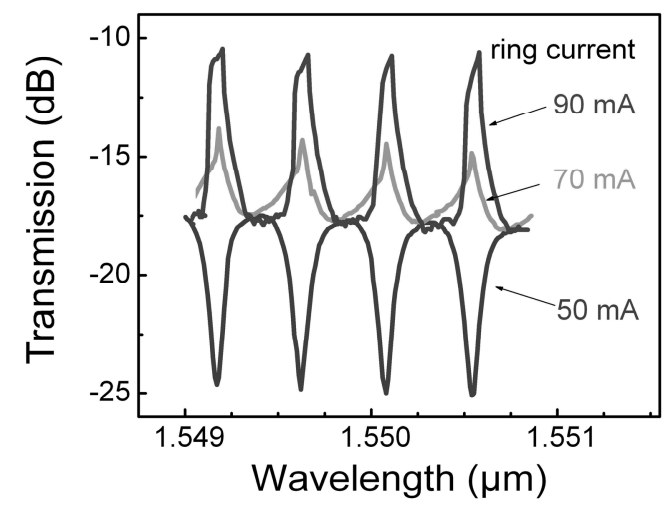

Fig. 8.26. Transmission characteristics of an active ring resonator (with SOAs) under different driving conditions, after [90].

Radiation losses as well as scattering losses due to sidewall roughness do essentially determine the residual loss in bent WGs [89], and they cannot be reduced easily, so that the losses may add up to an intolerable amount, if a larger number of rings is cascaded in order to get filter transmission characteristics, which are needed in many WDM applications (flat top, steep roll-off). One means to overcome these problems is the incorporation of active parts in the microrings, i. e. adding semiconductor optical amplifier (SOA) sections, which compensate WG losses. The effect of such SOAs is illustrated in Figs. 8.26:

At low drive currents of the SOAs the microring acts as a wavelength dependent filter with adjustable loss. If the current is increased so as to completely compensate for the losses within the ring, the structure gets an all-pass filter, and for even higher SOA currents the device becomes a ring laser.

Another application area of microrings, which is of general interest for fibre optic systems is all-optical wavelength conversion, since it can be made particularly efficient by an appropriately strong confinement of the optical waves within the microring [81,87]. Figure 8.27 shows corresponding results:

At present it is difficult to predict the future role of semiconductorbased microrings in fibre optic communication systems. Although microrings offer a number of attractive properties, they will always be in competition with alternative solutions, and competitiveness will in the end essentially be determined by the ratio of performance to cost including aspects like device-, subsystem-, operation-, and surveillance cost. 


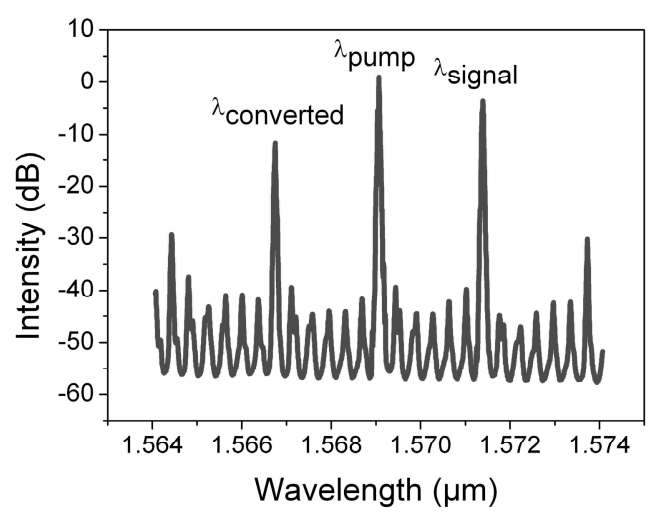

Fig. 8.27. All-optical wavelength conversion observed at the through-port of a microring resonator, after [87]

On the other hand, microrings are also finding increasing interest in areas beyond telecom, in particular for sensor applications, which includes semiconductor-based devices, but microrings in other material systems as well.

\subsection{All-optical Switching}

All-optical switching has been performed in GaAs/AlGaAs [91] and more recently in SOI micro rings [38] as well. The device structure under investigation has been a single micro ring coupled to a straight waveguide using either horizontal [91] or vertical [38] coupling. According to the considerations in Sect. 8.4. one may either switch from on-resonance to offresonance or the other way round by using any of the effects mentioned.

In the recently reported all-optical switching in SOI microrings [38] detuning is achieved by optical means, i.e. optically created free carriers modify the effective refractive index and provide a detuning of the MR resonances, which is equivalent to switching. These experiments have aroused a lot of speculation about the prospects and the potential of semiconductor/silicon-based all-optical signal processing. However, before devices of the type described here might find their way into real and widespread applications, a number of topics need additional investigations and significant improvements: The wavelength to be switched and the wavelength inducing the detuning must both be accurately adjusted to a ring resonance. High finesse of the resonances makes the switching efficient, but is demanding with respect to wavelength accuracy. On the other hand, 
relaxed wavelength tolerances (lower finesse) raise the required switching power significantly. The requirements become even more demanding due to polarisation effects. In addition, the free carriers needed for changing the refractive index have been created so far by two-photon absorption, and this process requires high photon densities, which in turn implies significant technological effort and cost. In the case of the SOI-ring based switching $10 \mathrm{ps}$ long pulses with $25 \mathrm{pJ}$ energy were used, generated by a Ti:sapphire picosecond laser in combination with an optical parametric oscillator. Finally, the observed relatively long exponential relaxation $\left(\tau_{\text {rel }}=450 \mathrm{ps}\right)$ of the SOI-device in combination with the short switching window of a few picoseconds is unfavourable for many application conditions. A better match between excitation and recovery time has been observed for the GaAs/AlGaAs microrings with about 50 ps recovery time, which is attributed to strong surface-state recombination at the microring sidewalls, but even in this case the switching window to recovery time ratio is far from optimum.

Thus the all-optical switching experiments are encouraging, but there is still a very long way to go before all-optical signal processing might find its way into practical applications, which is true for III-V semiconductors and even more so for silicon-based optical switching, on the other hand the latter would have a tremendous economic impact.

\subsection{Future Trends}

Future development of microring resonators are expected to go into different directions. One trend will be towards multi-stage filters with application-specific tailored characteristics. Devices will be mass-produced in non-expensive material systems and consequently such filters are likely to find widespread applications.

Another trend will be towards MR-based devices, which combine active and passive microrings and which can thus perform more complex functionalities. This will include active-passive functionalities on a single chip, but different kinds of MRs may equally well be part of even more complex hybrid (sub-)systems. One corresponding example is shown in Fig. 8.28, which illustrates a hybrid optical transceiver, comprising active, polymer and inorganic electro-optical materials, and passive, thermally tuneable microrings as well $[92,93]$.

Point-to-point links play a predominant role in current optical networks, but wavelength routing will become more significant in future optical networks, and under these circumstances reconfigurable wavelength-routers 


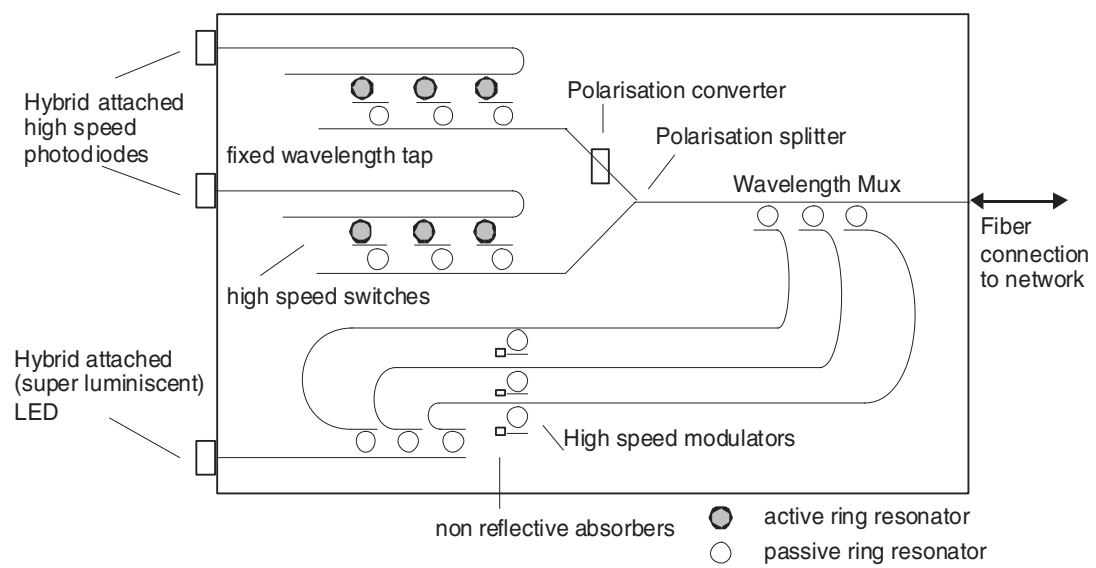

Fig. 8.28. Schematic overview of NAIS transceiver, were active (high speed modulators) and passive MRs are combined to integrate different functions well (after [94])

will become particularly relevant. Key features of such routers are wavelength splitting (and combining) plus wavelength routing, they will be used in wavelength-selective and broadcast applications as well, and they should be wavelength agile to a high degree. Figure 8.29 shows a corresponding router architecture based on MR integrated in the so-called Manhattan architecture. Corresponding devices are currently under investigation in the Netherlands within the project Broadband Photonics (BBPhotonics), funded by the Freeband communications program [95].

There is no doubt that MRs offer a large potential for a variety of applications, however, the ultimate success will depend essentially on the extent to what technical performance and price targets can be met simultaneously, which is true for all components in fibre optics.

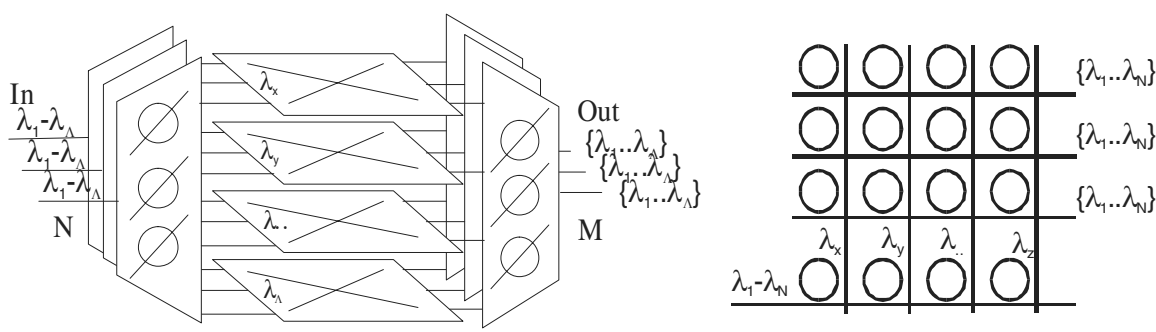

Fig. 8.29. Functional description of possible MR implementations (left) and schematic drawing of a MR based wavelength-router. 


\section{Acknowledgement}

The authors would like to thank the following persons for their contribution to this chapter: Edwin Klein, Dion Klunder, Freddy Tan, Manfred Hammer, Arne Leinse and Mart Diemeer, all from the University of Twente, The Netherlands, Dominik Rabus (Forschungszentrum Karlsruhe, Germany), and Brent E. Little (Little Optics Inc., Annapolis Junction, MD, USA).

\section{References}

1. A. Ashkin and J.M. Dziedzic: "Observation of optical resonances of dielectric spheres by light scattering," Appl. Opt. 20, 1803-1814 (1981).

2. H. B.Lin, A. L. Huston, B. L. Justus, and A. J. Campillo: "Some characteristics of a droplet whispering-gallery-mode laser," Opt. Lett. 11, 614-616 (1986)

3. S.C.Hill, D. H. Leach, and R. K. Chang: "Third-order sum-frequency generation in droplets: Model with numerical results for third- harmonic generation," J. Opt. Soc. Amer. B 10, 16-33 (1993)

4. M. M. Mazumder, S. C. Hill, D. Q. Chowdhury, and R. K. Chang: "Dispersive optical bistability in a dielectric sphere," J. Opt. Soc. Amer. B 12, 297-310 (1995)

5. J.Haavisto and G.A. Pajer: "Resonance effects in low-loss ring waveguides," Opt. Lett. 5, 510-512 (1980)

6. W. Weiershausen and R.Zengerle: "Photonic highway switches based on ring resonators used as frequency-selective components," Appl. Opt. 35, 5967-5978 (1996)

7. B.E. Little, S. T.Chu, W.Pan, and Y. Kokubun: "Microring resonator arrays for VLSI photonics," IEEE Photon. Technol. Lett. 12, 323-325 (2000)

8. S. Suzuki, K. Shuto, and Y.Hibino: "Integrated-optic ring resonators with two stacked layers of silica waveguide on Si," IEEE Photon. Technol. Lett. 4, 12561258 (1992)

9. J.H.Zhao and C.K. Madsen: Optical Filter Design and Analysis (Wiley, New York, 1999).

10. A. Driessen, D. H. Geuzebroek, H. J.W. M. Hoekstra, H. Kelderman, E. J. Klein, D. J. W. Klunder, C. G. H. Roeloffzen, F. Susanto, E. Krioukov, C. Otto, H. Gersen, N.F. van Hulst, and L. Kuipers: "Microresonators as building blocks for VLSI photonics," AIP Conf. Proc. 709 (F. Michelotti, A. Driessen, and M. Bertolotti, eds.), 1-18 (2003)

11. F.C.Blom, D. R.van Dijk, H.J.W.M.Hoekstra, A. Driessen, and T. J. A. Popma, "Experimental study of integrated-optics microcavity resonators: Toward an alloptical switching device," Appl. Phys. Lett. 71, 747-749 (1997)

12. E. J.Klein, D. H. Geuzebroek, H. Kelderman, and A. Driessen: "Wavelengthselective switch using thermally tunable microring resonators," Proc. IEEE Laser\&Electro-Optics Soc. Annual Meeting (LEOS 2003) (Tucson, AZ, USA, 2003) paper MM1 (2003)

13. J. T. Verdeyen: Laser Electronics (Prentice Hall, New Jersey, 1995) 
14. H. Kogelnik: "Theory of optical waveguides" in Guided-Wave Optoelectronics (T. Tamir, ed.), Chap. 2 (Springer, Heidelberg, Berlin, 1988)

15. B.E. Little and S. T.Chu: "Theory of polarization rotation and conversion in vertically coupled microresonators," IEEE Photon. Technol. Lett. 12, 401-403 (2000)

16. M.C.Larciprete, E. J.Klein, A. Belardini, D. H. Geuzebroek, A. Driessen, and F. Michelotti: "Polarization conversion in vertically coupled $\mathrm{Si}_{3} \mathrm{~N}_{4} / \mathrm{SiO}_{2}$ microring resonators", AIP Conf. Proc. 709 (F. Michelotti, A. Driessen, and M. Bertolotti, eds.) 415-416 (2003)

17. A. Melloni, F. Morichetti, and M. Martinelli: "Polarization conversion in ring resonator phase shifters," Optics Lett. 29, 2785-2787 (2004)

18. S.S.A. Obayya, B. M. A.Rahman, K. T.V.Grattan, and H. A.El-Mikati.: "Beam propagation modeling of polarization rotation in deeply etched semiconductor bent waveguides," IEEE Photon. Technol. Lett. 13, 681-683 (2001)

19. K. Wörhoff, L. T.H. Hilderink, A.Driessen, and P.V.Lambeck: "Silicon oxinitride - a versatile material for integrated optics applications," J. Electrochem. Soc. 149, F85- F91 (2002)

20. B.E. Little, S. T. Chu, P.P. Absil， J. V. Hryniewicz， F. G. Johnson， F. Seiferth, D. Gill, V. Van, O. King, and M. Trakalo: "Very high order microring resonator filters for WDM application," IEEE Photon. Technol. Lett. 16, 2263-2265 (2004)

21. Y.Kokubun, S. Kubota, and S. T. Chu: "Polarisation-independent vertically coupled microring resonator filter," Electron. Lett. 37, 90-92 (2001)

22. D. J.W. Klunder, C.G.H.Roeloffzen, and A.Driessen: "A novel polarizationindependent wavelength-division-multiplexing filter based on cylindrical microresonators,” IEEE J. Select. Topics Quantum Electron. 8, 1294-1299 (2002)

23. J. Capmany and M. A. Muriel: "A new transfer-matrix formalism for the analysis of fiber ring resonators - compound coupled structures for FDMA demultiplexing," J. Lightwave Technol. 8, 1904-1919 (1990).

24. O. Schwelb: "Transmission, group delay, and dispersion in single-ring optical resonators and add/drop filters - a tutorial overview," J. Lightwave Technol. 22, 1380-1394 (2004)

25. A. Vorckel, M. Mönster, W. Henschel, P. Haring Bolivar, and H. Kurz: "Asymmetrically coupled silicon-on-insulator microring resonators for compact adddrop multiplexers," IEEE Photon. Technol. Lett. 15, 921-923 (2003)

26. D. J. W. Klunder, E. Krioukov, F. S. Tan, T. van der Veen, H. F. Bulthuis, G. Sengo, C. Otto, H. J. W. M. Hoekstra, and A. Driessen: "Vertically and laterally waveguide-coupled cylindrical microresonators in $\mathrm{Si}_{3} \mathrm{~N}_{4}$ on $\mathrm{SiO}_{2}$ technology," Appl. Phys. B 73, 603-608 (2001)

27. K. Oda, N. Takato, and H. Toba: "A wide-FSR wave-guide double-ring resonator for optical FDM transmission-systems," J. Lightwave Technol. 9, 728-736 (1991)

28. S. Suzuki, K. Oda, and Y.Hibino: "Integrated-optic double-ring resonators with a wide free spectral range of $100 \mathrm{GHz}$," J. Lightwave Technol. 13, 1766-1771 (1995)

29. O. Schwelb: "A design for a high finesse parallel-coupled microring resonator filter," Microwave Opt. Technol. Lett. 38, 125-129 (2003)

30. C2V, Enschede, The Netherlands. www.c2v.nl 
31. G. Cusmai, F. Morichetti, P. Rosotti, R. Costa, and A. Melloni: "Circuit-oriented modelling of ring-resonators," Optics and Quantum Electron. 37, 343-358 (2005)

32. D.J.W. Klunder: Photon physics in integrated optics microresonators, (PhD Thesis, University of Twente, The Netherlands, 2002)

33. R. Grover, T. A. Ibrahim, T. N.Ding, Y.Leng, L.-C. Kuo, S. Kanakaraju, K. Amarnath, L.C.Calhoun, and P.-T.Ho: "Laterally coupled InP-based singlemode microracetrack notch filter," IEEE Photon. Technol. Lett. 15, 1082-1084 (2003)

34. S.C. Hagness, D. Rafizadeh, S. T. Ho, and A. Taflove: "FDTD microcavity simulations: Design and experimental realization of waveguide-coupled single-mode ring and whispering-gallery-mode disk resonators," J. Lightwave Technol. 15, 2154-2165 (1997)

35. M. B. J. Diemeer: "Polymeric thermo-optic space switches for optical communications," Opt. Materials 9, 192-200 (1998)

36. I. L. Gheorma and R. M. Osgood, Jr.: "Fundamental limitations of optical resonator based high-speed EO modulators," IEEE Photon. Technol. Lett. 14, 795-797 (2002)

37. T. A. Ibrahim, W.Cao, Y.Kim, J.Li, J.Goldhar, P.-T.Ho, and C.H.Lee: "Alloptical switching in a laterally coupled microring resonator by carier injection," IEEE Photon. Technol. Lett. 15, 36-38 (2003)

38. V.R. Almeida, C. A. Barrios, R. R. Panepucci, and M. Lipson: "All-optical control of light on a silicon chip," Nature 431, 1081-1084 (2004)

39. E. Krioukov, D. J. W. Klunder, A. Driessen, J. Greve and C. Otto: "Sensor based on an integrated optical microcavity," Opt. Lett, 27, 512-514 (2002)

40. E. Krioukov, D. J.W. Klunder, A.Driessen, J. Greve, and C. Otto: "Integrated optical microcavities for enhanced evanescent-wave spectroscopy," Opt. Lett. 27, 1504-1506 (2002)

41. D. H. Geuzebroek, E. J.Klein, H.Kelderman, and A Driessen: "Wavelength tuning and switching of a thermo-optic microring resonator," Proc. $11^{\text {th }}$ Europ. Conf Integr. Optics (ECIO’03), Prague, Czech Republic, 395-398 (2003)

42. P.Rabiei and W.H.Steier: "Tunable polymer double micro-ring filters," IEEE Photon. Technol. Lett. 15, 1255-1258 (2003)

43. M. B. J. Diemeer: "Organic and inorganic glasses for microring resonators," $A I P$ Conf. Proc. 709 (F. Michelotti, A. Driessen, and M. Bertolotti, eds.), 252-267 (2003)

44. P. Gunter: Nonlinear Optical Effects and Materials (Springer, Berlin, Heidelberg, 2000)

45. A. Leinse, M. B. J. Diemeer, A. Rousseau, and A. Driessen: "A novel high-speed polymeric eo modulator based on a combination of a microring resonator and an MZI,” IEEE Photon. Technol. Lett. 17, 2074-2076 (2005)

46. K. Djordjev, S. J. Choi, and P. D. Dapkus: "Active semiconductor microdisk devices," J. Lightwave Technol. 20, 105-113 (2002)

47. L. A. Coldren and S.W. Corzine: Diode lasers and photonic integrated circuits (Wiley Series in Microwave and Optical Engineering, Wiley, New York, 1995)

48. H.Haeiwa, T.Naganawa, and Y.Kokubun: "Wide range center wavelength trimming of vertically coupled microring resonator filter by direct UV irradiation to SiN ring core," IEEE Photon. Technol. Lett. 16, 135-137 (2004) 
49. R. Dekker, D. J.W. Klunder, A. Borreman, M. B. J. Diemeer, K. Wörhoff, A. Driessen, J. W. Stouwdam, and F. C. J. M. van Veggel: "Stimulated emission and optical gain in $\mathrm{LaF}_{3}$ : Nd nanoparticle-doped polymer based waveguides," Appl. Phys. Lett. 85, 6104-6106(2004)

50. D. G. Rabus: Realization of optical filters using ring resonators with integrated semiconductor optical amplifiers in GaInAsP/InP ( $\mathrm{PhD}$ Thesis, Berlin University of Technology, Germany, 2002)

51. M. C. Flemings, MR with MEMS, Sandia National Laboratory, www.sandia.gov

52. F. S. Tan: Integrated Optical Filters based on Microring Resonators, (PhD Thesis, University of Twente, The Netherlands, 2004)

53. D. Marcuse: Principles of Optical Fiber Measurements (Academic Press, New York, 1981)

54. D. H. Geuzebroek, E. J. Klein, H. Kelderman, N. Baker, and A. Driessen: "Compact wavelength-selective switch for gigabit filtering in access networks," IEEE Photon. Technol. Lett. 17, 336-338 (2005)

55. A. Küng, J.Budin, L. Thévenaz, and Ph.A. Robert: "Optical fiber ring resonator characterization by optical time-domain reflectometry," Opt. Lett. 22, 90-92 (1997)

56. J. V. Hryniewicz, P. P. Absil, B. E. Little, R. A. Wilson, and P.-T. Ho: "Higher order filter response in coupled microring resonators," IEEE Photon. Technol. Lett. 12, 320-322 (2000)

57. R. Orta, P. Savi, R. Tascone, and D. Trinchero: "Synthesis of multiple-ringresonator filters for optical systems," IEEE Photon. Technol. Lett. 7, 1447-1449 (1995)

58. D.G.Rabus, M.Hamacher, and H.Heidrich: "Resonance frequency tuning of a double ring resonator in GaInAsP/InP: Experiment and simulation," Jpn. J. Appl. Phys. 41, 1186-1189 (2002)

59. C. K. Madsen and J.H. Zhao: "A general planar waveguide autoregressive optical filter," J. Lightwave Technol. 14, 437-447 (1996)

60. B.E. Little, S. T. Chu, P.P. Absil, J. V.Hryniewicz, F. G. Johnson, F. Seiferth, D. Gill, V. Van, O. King, and M. Trakalo: "Very high-order microring resonator filters for WDM applications," IEEE Photon. Technol. Lett. 16, 2263-2265 (2004)

61. A. Melloni: "Synthesis of a parallel-coupled ring-resonator filter," Opt. Lett. 26, 917-919 (2001)

62. S. T. Chu, B. E. Little, W. G. Pan, T. Kaneko, and Y. Kokubun: "Second-order filter response from parallel coupled glass microring resonators," IEEE Photon. Technol. Lett. 11, 1426-1428 (1999)

63. G. Griffel: "Vernier effect in asymmetrical ring resonator arrays," IEEE Photon. Technol. Lett. 12, 1642-1644 (2000)

64. G. Griffel: "Synthesis of optical filters using ring resonator arrays," IEEE Photon. Technol. Lett. 12, 810-812 (2000)

65. R. Grover, V.Van, T. A. Ibrahim, P.P.Absil, L.C.Calhoun, F. G. Johnson, J. V.Hryniewicz, and P.-T.Ho: "Parallel-cascaded semiconductor microring resonators for high-order and wide-FSR filters," J. Lightwave Technol. 20, 900 905 (2002)

66. B. E. Little, S. T. Chu, J. V. Hryniewicz, and P.P. Absil: "Filter synthesis for periodically coupled microring resonators," Opt. Lett. 25, 344-346 (2000) 
67. C. J.Kaalund and G.Peng: "Pole-zero diagram approach to the design of ring resonator-based filters for photonic applications," J. Lightwave Technol. 22, 1548-1558 (2004)

68. Little Optics, USA, www.littleoptics.com / www.nomadics.com/

69. S. T. Chu, B.E. Little, W. G. Pan, T. Kaneko, S. Sato, and Y. Kokubun: "An eightchannel add-drop filter using vertically coupled microring resonators over a cross grid," IEEE Photon. Technol. Lett. 11, 691-693 (1999)

70. Z.Wang, W. Chen, and Y. J.Chen: "Unit cell design of crossbar switch matrix using micro-ring resonators," Proc. $30^{\text {th }}$ Europ. Conf. Opt. Commun. (ECOC'04), Stockholm, Sweden, Vol. 3, 462-463 (2004)

71. LioniX BV, Enschede, the Netherlands; www.lionixbv.nl

72. S. Suzuki, K. Shuto, and Y.Hibino: "Integrated-optic ring resonators with two stacked layers of silica waveguide on Si," IEEE Photon. Technol. Lett. 4, 12561259 (1992)

73. E. J. Klein, D.H Geuzebroek, H. Kelderman, G. Sengo, N. Baker, and A. Driessen: "Reconfigurable optical add-drop multiplexer using microring resonators," Proc. $12^{\text {th }}$ Europ. Conf. Integr. Optics (ECIO’05), Grenoble, France, 180-183 (2005)

74. D. H. Geuzebroek, E. J. Klein, H. Kelderman, C. Bornholdt, and A. Driessen: "40 Gbit/s reconfigurable optical add-drop multiplexer based on microring resonators," Proc. $31^{\text {st }}$ Europ. Conf. Opt. Commun. (ECOC'05), Glasgow, UK, 983$984(2005)$

75. Lambda Crossing, Israel, www.lambdax.com

76. J.Buus, D.J.Blumenthal, and M.-C. Amann: Tunable laser diodes and related optical sources, 2nd ed. (Wiley, New York, 2004)

77. Y. Yanagase, S. Suzuki, Y. Kokubun, and S. T. Chu: "Box-like filter response by vertically series coupled microring resonator filter," Proc. $27^{\text {th }}$ Europ. Conf. Opt. Commun. (ECOC'01), Amsterdam, NL, Vol. 4, 634-635 (2001)

78. K. Jinguji and M. Kawachi: "Synthesis of coherent two-port lattice-form optical delay-line circuit," J. Lightwave Technol. 13, 73-82 (1995)

79. G.-L. Bona, R. Germann, and B. J. Offrein: "SiON high-refractive-index waveguide and planar lightwave circuits," IBM J. Res. \& Dev. 47, 239-249 (2003)

80. G. L. Bona, F. Horst, R. Germann, B. J. Offrein, and D. Wiesmann: "Tunable dispersion compensator realized in high-refractive-index-contrast SiON technology," Proc. 28th Europ. Conf. Opt. Commun. (ECOC'02), Copenhagen, Denmark, Vol. 2, paper 4.2.1 (2002)

81. P.P. Absil, J. V. Hryniewicz， B. E. Little，P.S.Cho， R. A. Wilson， L. G. Joneckis, and P.-T.Ho: "Wavelength conversion in GaAs micro-ring resonators," Opt. Lett. 25, 554-556 (2000)

82. G. Griffel, J.H. Abeles, R. J.Menna, A.M. Braun, J.C.Connolly, and M. King: "Low-threshold InGaAsP ring lasers fabricated using bi-level dry etching," IEEE Photon. Technol. Lett. 12, 146-148 (2000)

83. R. Grover, P. Absil, V.Van, J.Hryniewicz, B. Little, O. King, L.Calhoun, F. Johnson, and P. Ho: "Vertically coupled GaInAsP InP microring resonators," Opt. Lett. 26, 506-508 (2001)

84. D. G. Rabus and M. Hamacher: "MMI coupled ring resonators in GaInAsP/InP," IEEE Photon. Technol. Lett. 13, 812-814 (2001) 
85. K. Djordjev, Seung-J. Choi, Sang-J.Choi, and P.D. Dapkus: "High-Q vertically coupled InP microdisk resonators," IEEE Photon. Technol. Lett. 14, 331-333 (2002)

86. K. Djordjev, Seung-J.Choi, Sang-J. Choi, and P.D. Dapkus: "Novel active switching components based on semiconductor microdisk resonators," Proc. $28^{\text {th }} \mathrm{Eu}$ rop. Conf. Opt. Commun. (ECOC'02), Copenhagen, Denmark, Vol. 1, paper 2.3.5 (2002)

87. M. Hamacher, U. Troppenz, H. Heidrich, and D. G. Rabus: "Active ring resonators based on InGaAsP/InP," Proc. SPIE Conf. Photonic Fabrication Europe, vol. 4947, 212-222 (2003)

88. Seung-J. Choi, Q. Yang, Z. Peng, Sang-J. Choi, and P. D. Dapkus: "High-Q buried heterostructure microring resonators," OSA Conf. Lasers and Electro Optics (CLEO 2004), San Francisco, CA, USA, paper CThF1 (2004)

89. Z.Bian, B.Liu, and A. Shakouri: "InP-based passive ring-resonator-coupled lasers," IEEE J. Quantum Electron. 39, 859-865 (2003)

90. U. Troppenz, M.Hamacher, D. G. Rabus, and H. Heidrich: "All-active InGaAsP/InP ring cavities for widespread functionalities in the wavelength domain," Proc. 14 $4^{\text {th }}$ Internat. Conf. Indium Phosphide and Related Materials (IPRM'02), Stockholm, Sweden, 475-478 (2002)

91. V.Van, T. A. Ibrahim, K. Ritter, P.P. Absil, F. G. Johnson, R. Grover, J. Goldhar, and P.-T. Ho: "All-optical nonlinear switching in GaAs-AlGaAs microring resonators," IEEE Photon. Technol. Lett. 14, 74-76 (2002)

92. P. Rabiei, W.H. Steier, C.Zhang, and L.R. Dalton: "Polymer micro-ring filters and modulators," J. Lightwave Technol. 20, 1968-1975 (2002).

93. P. Rabiei and W.H.Steier: "Tunable polymer double micro-ring filter," IEEE Photon. Technol. Lett. 15, 1255-1257 (2003).

94. NAIS, Next-generation Active Integrated-optic Subsystems IST-2000-28018

95. Freeband Communications, "Broadband Photonics" www.freeband.nl 
